NASA Technical Memorandum 106068

\title{
A New Lagrangian Method for Three-Dimensional Steady Supersonic Flows
}

Ching-Yuen Loh and Meng-Sing Liou

Lewis Research Center

Cleveland, Ohio

September 1993

\section{N/SA}




\title{
A NEW LAGRANGIAN METHOD FOR THREE-DIMENSIONAL STEADY SUPERSONIC FLOWS
}

\author{
Ching-Yuen Loh* and Meng-Sing Liou \\ National Aeronautics and Space Administration \\ Lewis Research Center \\ Cleveland, Ohio 44135
}

\section{SUMMARY}

In this report, the new Lagrangian method introduced by Loh and Hui is extended for three-dimensional, steady supersonic flow computation. The derivation of the conservation form and the solution of the local Riemann solver using the Godunov and the high-resolution TVD (total variation diminished) schemes is presented. This new approach is accurate and robust, capable of handling complicated geometry and interactions between discontinuous waves. Test problems show that the extended Lagrangian method retains all the advantages of the two-dimensional method (e.g., crisp resolution of a slip-surface (contact discontinuity) and automatic grid generation). In this report, we also suggest a novel three-dimensional Riemann problem in which interesting and intricate flow features are present.

\section{INTRODUCTION}

It is well known that there are two formulations describing fluid motion: the Eulerian and the Lagrangian. The inviscid compressible flow, as modeled by the Euler equations of gas dynamics, is of both theoretical and practical importance. Over the past four decades much progress has been made in its numerical simulation. Particularly in the 1980 's, we witnessed an exhaustive exploration of upwind, monotone schemes, especially with respect to exact and approximate Riemann solvers (see extensive review by Roe (ref. 1)). However, much of the current research, except that on one-dimensional flow, is based on the Eulerian description of fluid motion. In the 1950's and 1960's, studies of fluid motion based on the conventional Lagrangian description were performed, most notably in the Los Alamos and the Lawrence Livermore National Laboratories. One feature of the Lagrangian approach is that the computational grid is embedded in the fluid and distorted by its motion. This approach is limited by its inability to cope with a large distortion of the grid when it becomes tangled and highly irregular. Thus, a hybrid Lagrangian-Eulerian approach was attempted (Arbitrary Lagrangian Eulerian (ALE) method (ref. 2)) to recover the grid regularity. Unfortunately, the continuous geometrical interpolation in this hybrid approach eventually leads to loss of accuracy. As a result, since the late sixties, the Eulerian approach is favored for its easy control of grid and grid regularity. Even so, the very essence of the Lagrangian approach, that a computational cell is a fluid particle and remains intact, is missed in the Eulerian method. In the numerical simulation based on the Eulerian description, a slip-surface (contact discontinuity) that is linearly degenerated, is increasingly smeared as the solution marches further either in time or in space. The resolution of contact discontinuity in the Eulerian formulation is still a current research topic (e.g., see Harten (ref. 3)).

Recently, based on the concept of "Lagrangian time" introduced by Hui and Van Roessel (ref. 5), Loh and Hui (ref. 4) derived a new Lagrangian conservation form for the two-dimensional, inviscid compressible flow governed by Euler equations and successfully demonstrated its capability in supersonic flow computation. In this new formulation, the Lagrangian time $\tau$ and the stream function $\xi$ replace $x$ and $y$ as the independent variables and the remapping stage is eliminated. Loh and Hui introduced "geometrical conservation" to overcome the loss of accuracy in geometrical quantities. In the new Lagrangian formulation, a computational cell is literally a fluid particle and flow physics is closely followed. As a result, slipline (contact discontinuity) is crisply resolved, without the need for detection or artificial treatment, and is never further smeared.

\footnotetext{
*National Research Council-NASA Research Associate.
} 
In this report, we first extend the new Lagrangian approach of reference 4 to three-dimensional, steady supersonic flow computation. This is not a trivial extension for the geometry is complicated and the exact Riemann solution in multidimensions is not yet known. Therefore, we offer an approximate approach to the Riemann problem of the present Lagrangian formulation. In the next section, starting with the three-dimensional Eulerian conservation form and with the compatibility equations (the "geometrical conservation laws"), we introduce the new Lagrangian conservation form for three-dimensional steady flow. We then describe the implementation of the Godunov and TVD schemes and illustrate the so-called pseudo-three-dimensional Riemann problem and its solution. Next, we briefly discuss the well-posedness of the Cauchy problem in question. The well-posedness (the general CFL condition) controls the stability of the numerical procedure. We show how this condition is easily met in the present Lagrangian approach. Through several test examples in the section Test Problems, we demonstrate the robustness and accuracy of the new approach.

\section{THE NEW LAGRANGIAN CONSERVATION FORM FOR THREE-DIMENSIONAL STEADY FLOWS}

For any modern shock-capturing scheme, an appropriate conservation form is essential for accuracy. We begin the derivation of the Lagrangian conservation form with the Eulerian conservation laws written for threedimensional steady flows:

$$
\frac{\partial \mathbf{E}}{\partial x}+\frac{\partial \mathbf{F}}{\partial y}+\frac{\partial \mathbf{G}}{\partial z}=0
$$

where

$$
\mathbf{E}=\left(\begin{array}{c}
\rho u \\
\rho u^{2}+p \\
\rho u v \\
\rho u w \\
\rho u H
\end{array}\right) \quad \mathbf{F}=\left(\begin{array}{c}
\rho v \\
\rho v u \\
\rho v^{2}+p \\
\rho v w \\
\rho v H
\end{array}\right) \quad \mathbf{G}=\left(\begin{array}{c}
\rho w \\
\rho w u \\
\rho w v \\
\rho w^{2}+p \\
\rho w H
\end{array}\right)
$$

and $u, v$, and $w$ are the Cartesian components of flow velocity, $\rho$ is density, and $p$ is pressure of the fluid. The total enthalpy

$$
H=\frac{1}{2}\left(u^{2}+v^{2}+w^{2}\right)+\frac{\gamma}{\gamma-1} \frac{p}{\rho} \gamma=1.4
$$

Recently, Loh and Hui (ref. 4) introduced a new Lagrangian conservation form for two-dimensional steady supersonic flow computation, which is based on the concept of Lagrangian time (refs. 5 and 6). The Lagrangian time $\tau$ is a physical time: the time of motion of each fluid particle along its own streamline. For each fluid particle following its streamline, the Lagrangian time differs from the physical time $t$ only by a constant $t_{0}$

$$
\tau=t+t_{0}
$$


which can be considered the local initial time associated with each fluid particle. In this formulation, $\tau$ and the stream function $\xi$ replace $x$ and $y$ as the independent variables. A computational cell is literally a fluid particle and the flow physics is closely followed. The Lagrangian approach possesses many attractive features that are missed in the Eulerian approach, such as crisp resolution of slipline and automatic grid generation.

To explore a Lagrangian approach for higher dimensions, we extended the new Lagrangian formulation of reference 4 to a three-dimensional formulation and derived the corresponding conservation form. There are two types of the Lagrangian conservation form (Hui and Zhao, 1993, SIAM J. Sci. Comput., to be published): the primary one is based on Lagrangian time $\tau$, and the enhanced one is based on the Lagrangian distance $\lambda$, the distance (arc length) along a streamline. In the presence of strong contact discontinuities (slip-surfaces), the $\lambda$ conservation form performs better in numerical computations and is used in this report.

A three-dimensional, nonconservative Lagrangian formulation of the Euler equations of an inviscid, nonheatconducting, perfect gas, obeying the $\gamma$-law, has been described in references 4 and 5 . Here, starting from the Eulerian conservation form (1), we derive the corresponding new Lagrangian $\lambda$ conservation form with $\tau$ as an intermediate variable.

In a three-dimensional steady flow, there are two independent stream functions, say, $\xi$ and $\eta$. Each fixed $\xi$ or $\eta$ represents a stream surface (fig. 1(a)). A fixed pair of $\xi$ and $\eta$ denotes a streamline in the three-dimensional space. Following the streamline, the Lagrangian time $\tau$ or the Lagrangian distance $\lambda$ uniquely determines the location of the fluid particle. For example, $(\tau, \xi, \eta)$ can be considered a new set of independent variables that are now functions of the Cartesian coordinates $\mathbf{r}=(x, y, z)^{T}$ (where superscript $T$ denotes transposition). Since equation (2) holds along a streamline (on which $\xi$ and $\eta$ are fixed), the fluid velocity $\mathbf{V}$ is

$$
\mathbf{V}=(u, v, w)^{T}=\frac{\partial \mathbf{r}}{\partial t}=\frac{\partial \mathbf{r}}{\partial \tau}
$$

Furthermore, the following Lagrangian quantities are defined as

$$
\mathbf{T}=(U, V, W)^{T}=\frac{\partial \mathbf{r}}{\partial \xi} \quad \mathbf{S}=(X, Y, Z)^{T}=\frac{\partial \mathbf{r}}{\partial \eta}
$$

These quantities represent the lateral rate of displacement of a fluid particle (or computational cell). The determinant of the Jacobian $J$,

$$
|J|=\left|\frac{\partial(x, y, z)}{\partial(\tau, \xi, \eta)}\right|=\left|\begin{array}{ccc}
u & v & w \\
U & V & W \\
X & Y & Z
\end{array}\right|
$$

denotes the volume ratio during the change of independent variables. Subsequently, similar to reference 4,

$$
K=\rho|J|=\rho\left|\begin{array}{lll}
u & v & w \\
U & V & W \\
X & Y & Z
\end{array}\right|
$$

is the mass flux (fig. 1(d)). 
Note that the following relations are the compatibility conditions between the $\tau-, \xi_{-}$, and $\eta$-derivatives of $x, y$, and $z$ :

$$
\begin{aligned}
& \frac{\partial \mathbf{T}}{\partial \tau}=\frac{\partial \mathbf{V}}{\partial \xi} \\
& \frac{\partial \mathbf{S}}{\partial \tau}=\frac{\partial \mathbf{V}}{\partial \eta} \\
& \frac{\partial \mathbf{T}}{\partial \eta}=\frac{\partial \mathbf{S}}{\partial \xi}
\end{aligned}
$$

Starting with the Eulerian conservation form (1) and equations (3) to (7), the variable transformation from $(x, y, z)$ to $(\tau, \xi, \eta)$ achieves the conservation form based on Lagrangian time $\tau$. In this $\tau$ conservation form, each fluid particle marches forward with the same time step $\Delta \tau$ according to its own velocity. Across a contact discontinuity (slip-surface) where flow velocity may be discontinuous, two adjacent fluid particles initially in physical contact may eventually be separated from each other, rendering it difficult to apply a local Riemann solver in the Godunov or TVD schemes. A remedy is to keep these two particles marching at the same pace, or more generally, allow all the fluid particles to march the same distance $\Delta \lambda$ instead of the same time step $\Delta \tau$ along their own streamlines. This idea leads to another new Lagrangian conservation form - the conservation form based on the Lagrangian distance $\lambda$. We present here only a sketch of the necessary steps (see Hui and Zhao, 1993, SIAM J. Sci. Comput., to be published, for details).

First, we define the Lagrangian distance as

$$
\lambda=\int_{0}^{\tau} q d \tau
$$

where the flow speed

$$
q=\left(u^{2}+v^{2}+w^{2}\right)^{1 / 2}
$$

The other independent variables are the same as before:

$$
\left\{\begin{array}{l}
\xi_{1}=\xi \\
\eta_{1}=\eta
\end{array}\right.
$$

Let

$$
\alpha=\frac{\partial \lambda}{\partial \xi} \quad \beta=\frac{\partial \lambda}{\partial \eta}
$$

From equation (8), two useful relations can be easily derived:

$$
\frac{\partial \alpha}{\partial \lambda}=\frac{1}{q} \frac{\partial q}{\partial \xi} \quad \frac{\partial \beta}{\partial \lambda}=\frac{1}{q} \frac{\partial q}{\partial \eta}
$$


Making coordinate transformation from $\left(\lambda, \xi_{1}, \eta_{1}\right)$ to $(\tau, \xi, \eta)$, the Jacobian $J_{1}$ is

$$
J_{1}=\frac{\partial\left(\lambda, \xi_{1}, \eta_{1}\right)}{\partial(\tau, \xi, \eta)}=\left(\begin{array}{lll}
\partial \lambda / \partial \tau & \partial \xi_{1} / \partial \tau & \partial \eta_{1} / \partial \tau \\
\partial \lambda / \partial \xi & \partial \xi_{1} / \partial \xi & \partial \eta_{1} / \partial \xi \\
\partial \lambda / \partial \eta & \partial \xi_{1} / \partial \eta & \partial \eta_{1} / \partial \eta
\end{array}\right)=\left(\begin{array}{lll}
q & 0 & 0 \\
\alpha & 1 & 0 \\
\beta & 0 & 1
\end{array}\right)
$$

The inverted $J_{1}$ is

$$
J_{1}^{-1}=\frac{\partial(\tau, \xi, \eta)}{\partial\left(\lambda, \xi_{1}, \eta_{1}\right)}=\left(\begin{array}{lll}
\partial \tau / \partial \lambda & \partial \xi / \partial \lambda & \partial \eta / \partial \lambda \\
\partial \tau / \partial \xi_{1} & \partial \xi / \partial \xi_{1} & \partial \eta / \partial \xi_{1} \\
\partial \tau / \partial \eta_{1} & \partial \xi / \partial \eta_{1} & \partial \eta / \partial \eta_{1}
\end{array}\right)=\left(\begin{array}{ccc}
1 / q & 0 & 0 \\
-\alpha / q & 1 & 0 \\
-\beta / q & 0 & 1
\end{array}\right)
$$

Finally, a transformation from $(x, y, z)$ to $\left(\lambda, \xi_{1}, \eta_{1}\right)$ is achieved through successive coordinate transformations: $(x, y, z) \leftrightarrow(\tau, \xi, \eta) \leftrightarrow\left(\lambda, \xi_{1}, \eta_{1}\right)$. The resultant Jacobian $J_{0}$ is

$$
\begin{aligned}
J_{0}=\frac{\partial(x, y, z)}{\partial\left(\lambda, \xi_{1}, \eta_{1}\right)}= & \frac{\partial(\tau, \xi, \eta)}{\partial\left(\lambda, \xi_{1}, \eta_{1}\right)} \frac{\partial(x, y, z)}{\partial(\tau, \xi, \eta)}=\left(\begin{array}{ccc}
1 / q & 0 & 0 \\
-\alpha / q & 1 & 0 \\
-\beta / q & 0 & 1
\end{array}\right)\left(\begin{array}{ccc}
u & v & w \\
U & V & W \\
X & Y & Z
\end{array}\right) \\
& =\left(\begin{array}{ccc}
u / q & v / q & w / q \\
U-\alpha u / q & V-\alpha v / q & W-\alpha w / q \\
X-\beta u / q & Y-\beta v / q & Z-\beta w / q
\end{array}\right)
\end{aligned}
$$

Inverting the Jacobian $J_{0}$ gives

$$
J_{0}^{-1}=\frac{\partial\left(\lambda, \xi_{1}, \eta_{1}\right)}{\partial(x, y, z)}=\left(\begin{array}{lll}
\partial \lambda / \partial x & \partial \xi_{1} / \partial x & \partial \eta_{1} / \partial x \\
\partial \lambda / \partial y & \partial \xi_{1} / \partial y & \partial \eta_{1} / \partial y \\
\partial \lambda / \partial z & \partial \xi_{1} / \partial z & \partial \eta_{1} / \partial z
\end{array}\right)=\frac{q \rho}{K}\left(\begin{array}{lll}
K_{11} & K_{21} & K_{31} \\
K_{12} & K_{22} & K_{32} \\
K_{13} & K_{23} & K_{33}
\end{array}\right)
$$

Here, $K_{i, j}(i, j=1,2,3)$ are the cofactors of the determinant $\left|J_{0}\right|$. Note also that the determinant

$$
\left|J_{0}\right|=\frac{|J|}{q}=\frac{K}{p q}
$$

Equation (10) provides all the partial derivatives $\partial / \partial x, \partial / \partial y, \partial / \partial z$ that are needed in converting the Eulerian conservation form (1) into a new Lagrangian conservation form with $\left(\lambda, \xi_{1}, \eta_{1}\right)$ as the independent variables:

$$
\frac{\partial}{\partial x}=\frac{\rho q}{K}\left(K_{11} \frac{\partial}{\partial \lambda}+K_{21} \frac{\partial}{\partial \xi_{1}}+K_{31} \frac{\partial}{\partial \eta_{1}}\right)
$$




$$
\begin{aligned}
& \frac{\partial}{\partial y}=\frac{\rho q}{K}\left(K_{12} \frac{\partial}{\partial \lambda}+K_{22} \frac{\partial}{\partial \xi_{1}}+K_{32} \frac{\partial}{\partial \eta_{1}}\right) \\
& \frac{\partial}{\partial z}=\frac{\rho q}{K}\left(K_{13} \frac{\partial}{\partial \lambda}+K_{23} \frac{\partial}{\partial \xi_{1}}+K_{33} \frac{\partial}{\partial \eta_{1}}\right)
\end{aligned}
$$

After some algebraic manipulation, (1) is transformed into a new Lagrangian $\lambda$ conservation form:

$$
\frac{\partial}{\partial \lambda}\left(\begin{array}{c}
K \\
K u+p K_{11} \\
K v+p K_{12} \\
K w+p K_{13} \\
H K
\end{array}\right)+\frac{\partial}{\partial \xi_{1}}\left(\begin{array}{c}
0 \\
p K_{21} \\
p K_{22} \\
p K_{23} \\
0
\end{array}\right)+\frac{\partial}{\partial \eta_{1}}\left(\begin{array}{c}
0 \\
p K_{31} \\
p K_{32} \\
p K_{33} \\
0
\end{array}\right)=0
$$

Note that the first equation of (11) implies $K=$ constant along a streamline and therefore, the fifth equation of (11) is reduced to

$$
\frac{\partial H}{\partial \tau}=0
$$

Moreover, corresponding to equation (4), we define $\mathbf{T}_{1}$ and $\mathbf{S}_{1}$ as the line vectors of the Jacobian $J_{0}$, that is,

$$
\left\{\begin{array} { l } 
{ \mathbf { T } _ { \mathbf { 1 } } = \mathbf { T } - \alpha \mathbf { V } / q } \\
{ \mathbf { S } _ { \mathbf { 1 } } = \mathbf { S } - \beta \mathbf { V } / q }
\end{array} \quad \left\{\begin{array}{l}
\mathbf{T}_{\mathbf{1}}=\left(U_{1}, V_{1}, W_{1}\right)^{T} \\
\mathbf{S}_{\mathbf{1}}=\left(X_{1}, Y_{1}, Z_{1}\right)^{T}
\end{array}\right.\right.
$$

These vectors represent the geometrical deformation of the computational cells (fluid particles). Similar to the compatibility equations in (7), it can be shown, by using equations (7) and (9), that

$$
\begin{aligned}
& \frac{\partial \mathbf{T}_{\mathbf{1}}}{\partial \lambda}=\frac{\partial \mathbf{V} / q}{\partial \xi_{1}} \\
& \frac{\partial \mathbf{S}_{\mathbf{1}}}{\partial \lambda}=\frac{\partial \mathbf{V} / q}{\partial \eta_{1}} \\
& \frac{\partial \mathbf{T}_{1}}{\partial \eta_{1}}=\frac{\partial \mathbf{S}_{1}}{\partial \xi_{1}}
\end{aligned}
$$


By combining (11) and (12) and dropping the subscript 1 , we achieve a complete set of the new Lagrangian conservation form based on the $\lambda$-variable:

$$
\frac{\partial \mathbf{E}}{\partial \lambda}+\frac{\partial \mathbf{F}}{\partial \xi}+\frac{\partial \mathbf{G}}{\partial \eta}=0
$$

where

$$
\mathbf{E}=\left(\begin{array}{c}
e_{1} \\
e_{2} \\
e_{3} \\
e_{4} \\
e_{5} \\
e_{6} \\
e_{7} \\
e_{8} \\
e_{9} \\
e_{10} \\
e_{11} \\
e_{12} \\
e_{13} \\
e_{14}
\end{array}\right) \equiv\left(\begin{array}{c}
K \\
K u+p K_{11} \\
K v+p K_{12} \\
K w+p K_{13} \\
U \\
V \\
W \\
X \\
Y \\
Z \\
0 \\
0 \\
0
\end{array}\right) \mathbf{F}=\left(\begin{array}{c}
0 \\
0 \\
p K_{21} \\
p K_{22} \\
p K_{23} \\
-u / q \\
-v / q \\
-w / q \\
0 \\
0 \\
0 \\
X \\
Y \\
Z
\end{array}\right) \quad \mathbf{G}=\left(\begin{array}{c}
0 \\
0 \\
p K_{31} \\
p K_{32} \\
p K_{33} \\
0 \\
0 \\
0 \\
-u / q \\
-v / q \\
-w / q \\
-U \\
-V \\
-W
\end{array}\right)
$$

The system of Lagrangian conservation form (13) may look overwhelming at first glance and could be rejected prematurely. However, further examination reveals many simplifications and identities. The first two equations of (13) simply imply that $H=$ constant and $K=$ constant along a streamline; whereas the last three equations of (13) (i.e., the third vector equation of (12)) are shown automatically satisfied. For steady supersonic flows, one need only handle the remaining nine equations to march forward and solve the system. The six compatibility equations for $e_{6}, e_{7}, \ldots, e_{11}$ (components of $\mathbf{E}$ ) can be solved straightforwardly, as shown in the following section, and only the three momentum equations for $e_{3}, e_{4}$, and $e_{5}$ require more attention in the numerical procedure.

\section{APPLICATION OF GODUNOV AND TVD SCHEMES}

For a supersonic flow with the overall Mach number $M>1$ everywhere in the flow field, the system (13) is of hyperbolic type. In the past four decades, various numerical methods have been developed to handle the hyperbolic systems. There is a complete spectrum of shock-capturing, finite difference/finite volume schemes to solve the hyperbolic system of conservation laws, such as Godunov, flux-splitting, TVD, UNO, ENO, and other schemes. In this report, as an early exploration of the new three-dimensional Lagrangian method, we apply the basic Godunov scheme and then upgrade it to a high-resolution TVD scheme by means of Sweby's flux limiter (ref. 8). 


\section{Application of Godunov Scheme}

The physical and computational domains in the $(\lambda, \xi, \eta)$ space are illustrated, respectively, in figure 1, parts (a) and (b). A cuboid mesh in the computational domain is used and the computation marches in Lagrangian distance $\lambda$. The superscript $k$ refers to the marching step number and the subscripts $i$ and $j$ refer to the cell number on the distance plane ( $\lambda$-plane with $\lambda=$ constant) (fig. 1(c)). The marching step $\Delta \lambda^{k}=\lambda^{k+1}-\lambda^{k}$ is uniform for all $i$ and $j$. It may vary with $k$ but is always chosen to satisfy the usual CFL linear stability condition. The mesh divides the computational domain into cuboid control volumes or cells that are centered at $\left(\tau^{k}, \xi_{i}, \eta_{j}\right)$ in $\xi$ - and $\eta$-directions and have heights $\Delta \xi_{i}=\xi_{i+1 / 2}-\xi_{i-1 / 2}$ and $\Delta \eta_{j}=\eta_{j+1 / 2}-\eta_{j-1 / 2}$ (for all $k$ ). Unless otherwise stated, we use a uniform cell width $\Delta \xi_{i}$ for all $i$ and $\Delta \eta_{j}$ for all $j$.

In the physical space, a cuboid cell marching in $(\lambda, \xi, \eta)$ space corresponds to a fluid particle marching along its stream tube with step $\Delta \lambda$. The fluid particle is bounded by four stream surfaces $\xi=\xi_{i \pm 1 / 2}$ and $\eta=\eta_{j \pm 1 / 2}$ around it (fig. 1(c)). The $\xi \eta$-plane in computational space corresponds to the initial surface in the physical space. Any curvilinear coordinate mesh on the initial surface can be used as the $\xi \eta$-coordinate mesh and the initial $\mathbf{T}$ and $\mathbf{S}$ can be determined as part of the initial condition. A solid wall is always a stream surface and therefore, a coordinate surface.

The finite difference scheme of Godunov (ref. 8) for system (13) is derived by applying the divergence theorem to the cuboid cell $(i, j, k)$. The result is

$$
\begin{gathered}
\mathbf{E}_{i, j}^{k+1}=\mathbf{E}_{i, j}^{k}-\frac{\Delta \lambda^{k}}{\Delta \xi_{i}}\left(\mathbf{F}_{i+1 / 2, j}^{k+1 / 2}-\mathbf{F}_{i-1 / 2, j}^{k+1 / 2}\right)-\frac{\Delta \lambda^{k}}{\Delta \eta_{j}}\left(\mathbf{G}_{i, j+1 / 2}^{k+1 / 2}-\mathbf{G}_{i, j-1 / 2}^{k+1 / 2}\right) \\
i=1,2, \ldots, m \quad j=1,2, \ldots, n
\end{gathered}
$$

where the notation for the cell average of any quantity $f$ is

$$
f_{i, j}^{k}=\frac{1}{\Delta \xi_{i} \Delta \eta_{j}} \int_{\xi_{i-1 / 2}}^{\xi_{i+1 / 2}} f\left(\lambda^{k}, \xi, \eta\right) d \xi d \eta
$$

and the notation for $\lambda$ average of $f$ is

$$
\begin{aligned}
& f_{i+1 / 2, j}^{k+1 / 2}=\frac{1}{\Delta \lambda^{k}} \int_{\lambda^{k}}^{\lambda^{k+1}} f\left(\lambda, \xi_{i+1 / 2}, \eta_{j}\right) d \lambda \\
& f_{i, j+1 / 2}^{k+1 / 2}=\frac{1}{\Delta \lambda^{k}} \int_{\lambda^{k}}^{\lambda^{k+1}} f\left(\lambda, \xi_{i}, \eta_{j+1 / 2}\right) d \lambda
\end{aligned}
$$

In equation (14) the cell-interface fluxes $\mathbf{F}_{i+1 / 2, j}^{k+1 / 2}$ and $\mathbf{G}_{i, j+1 / 2}^{k+1 / 2}$ for the cell $(i, j)$ should be obtained from the self-similar solution of a local three-dimensional Riemann problem formed by the average constant state $\mathbf{Q}_{i, j}=(u, v, w, p, \rho)_{i, j}^{T}$ of the cell $(i, j)$ and those of its adjacent cells (fig. 2(a)). Unfortunately, as a result of its complexity, the exact solution to a general three-dimensional Riemann problem is not yet available (Chang and Hsiao (ref. 9)). However, it is known that a monotone difference scheme applied to a general conservation form converges to the physically relevant entropy-satisfying solution (see Harten et al. (ref 10)). In particular, 
Crandall and Majda (ref. 11) established the rigorous convergence for dimensional-splitting algorithms when each step is approximated by a monotone difference scheme (such as the Godunov scheme) for a single conservation law of multidimension. We extended and applied the dimensional splitting in the Godunov scheme for our hyperbolic system of conservation laws (13).

When applying the dimension-splitting technique in the Godunov scheme for (13), one need only solve pseudo-three-dimensional Riemann problems formed by two adjacent constant states, say, $\mathbf{Q}_{i, j}$ and $\mathbf{Q}_{i+1, j}$ instead of genuine three-dimensional ones (figs. 2(a) and (b)). In fact, a pseudo-three-dimensional Riemann problem is identical to a two-dimensional problem (ref. 4) except that the direction of the interaction line of the two constant states must be known. An interaction line is the line at which the 2 three-dimensional flows begin to contact and interact with each other. More detail is given about the pseudo-three-dimensional Riemann problem in the following section. To find the direction or the unit vector of the interaction line and solve the corresponding pseudo-three-dimensional Riemann problem, and for which we are given cells $(i, j)$ and $(i+1, j),\left(\mathbf{Q}_{i, j}, \mathbf{T}_{i, j}, \mathbf{S}_{i, j}\right)$, and $\left(\mathbf{Q}_{i+1, j}, \mathbf{T}_{i+1, j}, \mathbf{S}_{i+1, j}\right)$, the following steps are followed (fig. 2(c)):

(1) Calculate the unit normal vectors of the $\lambda$ surface of cells $(i, j)$ and $(i+1, j)$ :

$$
\mathbf{n}_{i, j}=\frac{\mathbf{T}_{i, j} \times \mathbf{S}_{i, j}}{\left|\mathbf{T}_{i, j} \times \mathbf{S}_{i, j}\right|} \quad \mathbf{n}_{i+1, j}=\frac{\mathbf{T}_{i+1, j} \times \mathbf{S}_{i+1, j}}{\left|\mathbf{T}_{i+1, j} \times \mathbf{S}_{i+1, j}\right|}
$$

If the cell is a boundary cell, that is, one or more of its walls is a given solid body surface and interacts with the solid boundary (fig. 2(d)), we still calculate the unit normal corresponding to the cell $(i, j)$ :

$$
\mathbf{n}_{i, j}=\frac{\mathbf{T}_{i, j} \times \mathbf{S}_{i, j}}{\left|\mathbf{T}_{i, j} \times \mathbf{S}_{i, j}\right|}
$$

(2) Calculate $\mathbf{m}_{1}=\mathbf{n}_{i, j} \times \mathbf{n}_{i+1, j}$, if $\left|\mathbf{m}_{1}\right|<\varepsilon$, where $\boldsymbol{\varepsilon}$ is a threshold value to avoid possible illconditionedness and filter possible noise; discard the present $\mathbf{m}_{1}$ and use the one of the previous time step. Then $\mathbf{m}_{1}$ is normalized to a unit vector. If a solid wall is present,

$$
\mathbf{m}_{1}=\mathbf{n}_{\text {wall }} \times \mathbf{n}_{i, j}
$$

Here we assume the unit normal vector $\mathbf{n}_{\text {wall }}$ of the solid body surface is given as a boundary condition. On the initial surface, $\mathbf{m}_{1}$ is specified as the unit vector of cell interface lines.

(3) Project the flow velocity vector $\mathbf{V}_{i, j}$ on the plane perpendicular to $\mathbf{m}_{1}$ (the interaction plane, fig. 2(e)) to obtain $\mathbf{V}_{b} ; \mathbf{V}_{b}$ is then normalized to give $\mathbf{m}_{2}$-the second unit vector, which is normal to $\mathbf{m}_{1}$ as well. Meanwhile, $\mathbf{V}_{i+1, j}$ is projected on the same interaction plane, yielding $\mathbf{V}_{t}$ :

$$
\left\{\begin{array}{l}
\mathbf{V}_{i, j}=\mathbf{V}_{b}+\mathbf{V}_{b 1} \\
\mathbf{v}_{i+1, j}=\mathbf{V}_{t}+\mathbf{V}_{t 1}
\end{array}\right.
$$


with

$$
\left\{\begin{array}{l}
\mathbf{v}_{b 1}=\left(\mathbf{V}_{i, j} \cdot \mathbf{m}_{1}\right) \mathbf{m}_{1} \\
\mathbf{V}_{t 1}=\left(\mathbf{V}_{i+1, j} \cdot \mathbf{m}_{1}\right) \mathbf{m}_{1}
\end{array}\right.
$$

where the subscripts $b$ and $t$ correspond to bottom and top states (see following section) which are the counterparts of right and left states in one-dimensional unsteady flow.

(4) Let $\mathbf{m}_{3}=\mathbf{m}_{1} \times \mathbf{m}_{2}$, then $\mathbf{m}_{1}, \mathbf{m}_{2}, \mathbf{m}_{3}$ form a local right hand Cartesian coordinate system. $\mathbf{m}_{2}$ and $\mathbf{m}_{3}$ span the interaction plane, in which we have two constant states:

$$
\left\{\begin{array}{l}
\mathbf{Q}_{b}=\left(u_{b}, v_{b}, p_{b}, \rho_{b}\right)^{T}=\left(\left|\mathbf{V}_{b}\right|, 0, p_{i, j}, \rho_{i, j}\right) \\
\mathbf{Q}_{t}=\left(u_{t}, v_{t}, p_{t}, \rho_{t}\right)^{T}=\left(\mathbf{V}_{t} \cdot \mathbf{m}_{2}, \mathbf{V}_{t} \cdot \mathbf{m}_{3}, p_{i+1, j}, \rho_{i+1, j}\right)
\end{array}\right.
$$

Then a standard two-dimensional Riemann solver (see next section), can be used to solve for the new $\mathbf{Q}_{b}$, and $\mathbf{Q}_{t}$ If the cell is a boundary cell interacting with the solid boundary, the data of $\mathbf{Q}=\mathbf{Q}_{t}$ is enough for computing new $\mathbf{Q}$ by means of a standard two-dimensional boundary Riemann solver.

(5) Recover new interface three-dimensional states for the purpose of computing cell interface fluxes:

$$
\mathbf{V}_{i+1 / 2, j}^{k+1 / 2}=u_{b} \mathbf{m}_{2}+v_{b} \mathbf{m}_{3}+\mathbf{V}_{b 1} \quad p_{i+1 / 2, j}^{k+1 / 2}=p_{b} \quad \rho_{i+1 / 2, j}^{k+1 / 2}=\rho_{b}
$$

The same procedure, steps (1) through (5), should be carried out for all four cell interfaces around the cell $(i, j)$, that is, the interfaces $(i \pm 1 / 2, j \pm 1 / 2)$ (fig. $1(\mathrm{c}))$.

In the first-order Godunov scheme, the cell-average $\mathbf{E}_{i, j}^{k}$ at time step $k$ is considered constant within cell $(i, j)$ and the fluxes $\mathbf{F}_{i+1 / 2, j}^{k+1 / 2}$ along the interface between cells $(i, j)$ and $(i+1, j)$ and $\mathbf{G}_{i, j+1 / 2}^{k+1 / 2}$ along the interface between cells $(i, j)$ and $(i, j+1)$ from step $k$ to step $k+1$ are obtained as in equations (16) and (17). Due to the self-similarity of the solution of the pseudo-three-dimensional Riemann problem, for any components $f$ of fluxes $\mathbf{F}$ and $\mathbf{G}$, equations (16) and (17) can be reduced to

$$
\begin{aligned}
& f_{i+1 / 2, j}^{k+1 / 2}=f\left(\tau^{k+1 / 2}, \xi_{i+1 / 2}, \eta_{j}\right) \\
& f_{i, j+1 / 2}^{k+1 / 2}=f\left(\tau^{k+1 / 2}, \xi_{i}, \eta_{j+1 / 2}\right)
\end{aligned}
$$

From (13), note that $f$ is a simple function of $\mathbf{Q}, \mathbf{T}$, and $\mathbf{S}$.

The complete numerical procedure of the Godunov method is summarized as follows:

(1) For initiation, given a three-dimensional flow problem in the Cartesian $(x, y, z)$ space, we choose a surface $\Gamma$, not itself a stream surface, on which the flow is known (e.g., a given uniform flow), as the initial surface $\lambda=0$ (fig. 1(a)). Then a parameterized curvilinear $\xi \eta$-coordinate mesh is laid on $\Gamma$ (for instance, $\xi$ and $\eta$ are equal to the arc length of their corresponding coordinate lines on $\Gamma$ ) with 


$$
\xi=\xi_{0}, \xi_{1}, \xi_{2}, \ldots, \xi_{m} \quad \eta=\eta_{0}, \eta_{1}, \eta_{2}, \ldots, \eta_{n}
$$

and $\xi_{0}$ or $\eta_{0}$ coinciding with the solid body surfaces (fig. 1). Therefore, $\mathbf{T}^{0}$ and $\mathbf{S}^{0}$ as well as the flow variable $\mathbf{Q}^{0}$ are known on $\Gamma$ as initial conditions. Then $\mathbf{E}_{i, j}^{0}$ are known on $\Gamma$ as well. In most of the test examples given in this report, the uniform free stream is the $x$-direction, the $y z$-plane is the initial surface $\Gamma$, and $\xi$ and $\eta$ are the respective arc lengths of $y$ - and $z$-coordinate lines. This results in $\mathbf{T}^{0}=(0,1,0)^{T}$ and $\mathbf{S}^{0}=(0,0,1)^{T}$ and the averaged $\mathbf{E}^{0}$ follows from equation (15) straightforwardly.

(2) With all $\mathbf{E}_{i, j}^{k}$ and $\mathbf{Q}_{i, j}^{k}$ known at step $k(k=0,1,2, \ldots)$, solve the local pseudo-three-dimensional Riemann problems (or local boundary Riemann problem) at the cell interfaces and obtain the cell interface flow variables: $\mathbf{V}_{i \pm 1 / 2, j \pm 1 / 2}^{k+1 / 2}, p_{i \pm 1 / 2, j \pm 1 / 2}^{k+1 / 2}$, and $\rho_{i \pm 1 / 2, j \pm 1 / 2}^{k+1 / 2}$ as described in the aforementioned context. Then fluxes $\mathbf{F}$ and $\mathbf{G}$ are calculated from equations (18) and (19), or more explicitly, from $\mathbf{F}$ and $\mathbf{G}$ expressions in (13). In order to do so, first update $\mathbf{T}_{i, j}^{k}$ and $\mathbf{S}_{i, j}^{k}$ to $\mathbf{T}_{i, j}^{k+1}$ and $\mathbf{S}_{i, j}^{k+1}$ :

$$
\begin{gathered}
\left(\begin{array}{c}
U_{i, j}^{k+1} \\
V_{i, j}^{k+1} \\
W_{i, j}^{k+1}
\end{array}\right)=\mathbf{T}_{i, j}^{k+1}=\mathbf{T}_{i, j}^{k}+\frac{\Delta \lambda^{k}}{\Delta \xi_{i}}\left(\frac{\mathbf{V}_{i+1 / 2, j}^{k+1 / 2}}{q_{i+1 / 2, j}^{k+1 / 2}}-\frac{\mathbf{V}_{i-1 / 2, j}^{k+1 / 2}}{q_{i-1 / 2, j}^{k+1 / 2}}\right) \\
\left(\begin{array}{c}
X_{i, j}^{k+1} \\
Y_{i, j}^{k+1} \\
Z_{i, j}^{k+1}
\end{array}\right)=\mathbf{S}_{i, j}^{k+1}=\mathbf{S}_{i, j}^{k}+\frac{\Delta \lambda^{k}}{\Delta \eta_{i}}\left(\frac{\mathbf{V}_{i, j+1 / 2}^{k+1 / 2}}{q_{i, j+1 / 2}^{k+1 / 2}}-\frac{\mathbf{V}_{i, j-1 / 2}^{k+1 / 2}}{q_{i, j-1 / 2}^{k+1 / 2}}\right) \\
i=1,2, \ldots, m \quad j=1,2, \ldots, m \quad k=0,1,2, \ldots
\end{gathered}
$$

With $(U, V, W)^{T}$ and $(X, Y, Z)^{T}$ from equations (20) and (21), the other components of the interface fluxes $\mathbf{F}$ and $\mathbf{G}$ are calculated according to their expressions in (13), by using the known cell-interface flow variables,

$\mathbf{V}_{i \pm 1 / 2, j \pm 1 / 2}^{k+1 / 2}$ and $p_{i \pm 1 / 2, j \pm 1 / 2}^{k+1 / 2}$.

(3) Use equation (14) to update $\mathbf{E}_{i, j}$ and advance one step. At this stage, one needs to update only $e_{3}, e_{4}$, and $e_{5}$. There is no need to update the constants $e_{1}, e_{2}, e_{12}, e_{13}$, and $e_{14}$; whereas $e_{6}, e_{7}, \ldots, e_{11}$, identical to $U$, $V, W, X, Y$, and $Z$, have been computed in the previous step.

(4) Decode $\mathbf{E}_{i, j}^{k+1}$ to get $\mathbf{Q}_{i, j}^{k+1}$. For simplicity, all the superscripts and subscripts are dropped off except those for E. Recall that

and define

$$
K_{11}=\left|\begin{array}{ll}
V & W \\
Y & Z
\end{array}\right| \quad K_{12}=-\left|\begin{array}{cc}
U & W \\
X & Z
\end{array}\right| \quad K_{13}=\left|\begin{array}{ll}
U & V \\
X & Y
\end{array}\right|
$$




$$
\begin{gathered}
A=\frac{1+\gamma}{1-\gamma}\left(K_{11}^{2}+K_{12}^{2}+K_{13}^{2}\right) \\
B=\frac{2}{1-\gamma}\left(K_{11} e_{3}+K_{12} e_{4}+K_{13} e_{5}\right) \quad C=e_{3}^{2}+e_{4}^{2}+e_{5}^{2}-2 K^{2} H
\end{gathered}
$$

Then the pressure $p$ satisfies the quadratic equation

$$
A p^{2}+B p+C=0
$$

It can be shown that $\Delta=B^{2}-4 A C \geq 0$ and the physically appropriate solution for $p$ is

$$
p=\frac{-B+\sqrt{\Delta}}{2 A}
$$

and the other flow variables are

$$
\begin{gathered}
u=\frac{e_{3}-p K_{11}}{K} \quad v=\frac{e_{4}-p K_{12}}{K} \quad w=\frac{e_{5}-p K_{13}}{K} \\
\rho=\frac{K}{\left(K_{11} u+K_{12} v+K_{13} w\right)}
\end{gathered}
$$

(5) Generate grid points (coordinates of cell centers) along streamlines and complete the procedure of marching forward by one step:

$$
\begin{gathered}
x_{i, j}^{k+1}=x_{i, j}^{k}+\frac{1}{2} \Delta \lambda^{k}\left(\frac{u_{i, j}^{k}}{q_{i, j}}+\frac{u_{i, j}^{k+1}}{q_{i, j}}\right) \\
y_{i, j}^{k+1}=y_{i, j}^{k}+\frac{1}{2} \Delta \lambda^{k}\left(\frac{v_{i, j}^{k}}{q_{i, j}}+\frac{v_{i, j}^{k+1}}{q_{i, j}}\right) \\
z_{i, j}^{k+1}=z_{i, j}^{k}+\frac{1}{2} \Delta \lambda^{k}\left(\frac{w_{i, j}^{k}}{q_{i, j}}+\frac{w_{i, j}^{k+1}}{q_{i, j}}\right) \\
i=1,2, \ldots, m \quad j=1,2, \ldots, n
\end{gathered}
$$

Step (5) is not a standard Godunov procedure but is unique to the Lagrangian method. By proceeding in this way, a three-dimensional mesh is automatically generated along the streamlines. Each grid point represents the center of a fluid particle (a computational cell). 
This completes the numerical procedure for one space step. To march forward further, repeat steps (2) through (5).

In the preceding Godunov procedure, the last three compatibility equations of (13) are simply ignored since they are already automatically satisfied and do not contribute to the marching forward of $\mathbf{E}$.

\section{Application of TVD Scheme}

It is well known that because of the presence of numerical viscosity, the first-order Godunov scheme strongly smears a discontinuity. To improve accuracy, various efforts have been made in the past decade to develop high-resolution montonicity-preserving schemes, such as TVD, ENO, and other schemes. Among these, Sweby's TVD scheme procedure (ref. 7), which adds limited antidiffusive terms to the first-order Gudonov scheme, is probably most convenient.

With the results from the Godunov scheme, the $\mathbf{E}$ vector is upgraded component by component before it is decoded to give new flow variables $\mathbf{Q}$ in the aforementioned Godunov procedure. Following Sweby (ref. 7), we apply a flux limiter function $\phi$ and rewrite (13) in the form

$$
\begin{aligned}
\mathbf{E}_{i, j}^{k+1}=\mathbf{E}_{i, j}^{k}-\mu \Delta_{-}^{\xi}[\mathbf{F} & \mathbf{G}]_{i+1 / 2, j}^{k+1 / 2}-\kappa \Delta_{-}^{\eta}[\mathbf{G} \mathbf{G}]_{i, j+1 / 2}^{k+1 / 2} \\
& -\mu \Delta_{-}^{\xi}\left\{\phi\left(r_{i}^{+}\right) \alpha_{i+1 / 2}^{+}\left[\Delta \mathbf{F}_{i+1 / 2, j}\right]^{+}-\phi\left(r_{i+1}^{-}\right) \alpha_{i+1 / 2}^{-}\left[\Delta \mathbf{F}_{i+1 / 2, j}\right]^{-}\right\} \\
& -\kappa \Delta_{-}^{\eta}\left\{\phi\left(s_{j}^{+}\right) \beta_{j+1 / 2}^{+}\left[\Delta \mathbf{G}_{i, j+1 / 2}\right]^{+}-\phi\left(s_{j+1}^{-}\right) \beta_{j+1 / 2}^{-}\left[\Delta \mathbf{G}_{i, j+1 / 2}\right]^{-}\right\}
\end{aligned}
$$

where $\mu=\Delta \lambda^{k} / \Delta \xi_{i}, \kappa=\Delta \lambda^{k} / \Delta \eta_{j}$, and the superscript $\mathbf{G}$ stands for the numerical fluxes of Godunov scheme. For any vector $\mathbf{H}$ the backward difference operators are

$$
\begin{aligned}
& \Delta_{-}^{\xi}\left[\mathbf{H}_{i+1 / 2, j}\right]=\mathbf{H}_{i+1 / 2, j}-\mathbf{H}_{i-1 / 2, j} \\
& \Delta_{-}^{\eta}\left[\mathbf{H}_{i, j+1 / 2}\right]=\mathbf{H}_{i, j+1 / 2}-\mathbf{H}_{i, j-1 / 2}
\end{aligned}
$$

The notation associated with the $\xi$-fluxes are

$$
\begin{gathered}
\left.\left[\Delta \mathbf{F}_{i+1 / 2, j}\right]^{+}=\mathbf{F}\left(\mathbf{Q}_{i+1, j}^{k}\right)-\mathbf{F}_{i+1 / 2, j}^{\mathbf{G}}{ }_{i+1 / 2}^{k+1 / 2, j}\right]^{-}=\mathbf{F}_{i+1 / 2, j}^{\mathbf{G}_{i+1 / 2}^{k+1}}-\mathbf{F}\left(\mathbf{Q}_{i, j}^{k}\right) \\
{\left[\Delta \mathbf{F}_{i+1}\right)} \\
\alpha_{i+1 / 2}^{ \pm}=\frac{1}{2}\left[1 \mp v_{i+1 / 2}^{ \pm}\right]
\end{gathered}
$$




$$
\begin{aligned}
& v_{i+1 / 2}^{ \pm}=\frac{\mu\left[\Delta\left(f_{Q}\right)_{i+1 / 2, j}\right]^{ \pm}}{\left[\left(e_{Q}\right)_{i+1, j}^{k}-\left(e_{Q}\right)_{i, j}^{k}\right]} \\
& r_{i}^{ \pm}=\left\{\left.\frac{\alpha_{i-1 / 2}^{ \pm}\left[\Delta\left(f_{Q}\right)_{i-1 / 2}\right]^{ \pm}}{\alpha_{i+1 / 2}^{ \pm}\left[\Delta\left(f_{Q}\right)_{i+1 / 2}\right]^{ \pm}}\right|^{ \pm 1}\right.
\end{aligned}
$$

Similarly for the $\eta$-fluxes, we have

$$
\begin{gathered}
{\left[\Delta \mathbf{G}_{i, j+1 / 2}\right]^{+}=\mathbf{G}\left(\mathbf{Q}_{i, j+1}^{k}\right)-\mathbf{G}_{i, j+1 / 2}^{\mathbf{G}} \begin{array}{c}
k+1 / 2 \\
{\left[\Delta \mathbf{G}_{i, j+1 / 2}\right]^{-}=\mathbf{G}_{i, j+1 / 2} \mathbf{G}_{i+1 / 2}^{k+\mathbf{G}}\left(\mathbf{Q}_{i, j}^{k}\right)}
\end{array}} \\
\beta_{j+1 / 2}^{ \pm}=\frac{1}{2}\left[1 \mp \omega_{j+1 / 2}^{ \pm}\right] \\
\omega_{j+1 / 2}^{ \pm}=\frac{\kappa\left[\Delta\left(g_{\ell}\right)_{i, j+1 / 2}\right]^{ \pm}}{\left[\left(e_{\ell}\right)_{i, j+1}^{k}-\left(e_{\ell}\right)_{i, j}^{k}\right]} \\
s_{j}^{ \pm}=\left\{\frac{\beta_{j-1 / 2}^{ \pm}\left[\Delta\left(g_{\ell}\right)_{j-1 / 2}\right]^{ \pm}}{\beta_{j+1 / 2}^{ \pm}\left[\Delta\left(g_{\ell}\right)_{j+1 / 2}\right]^{ \pm}}\right\}
\end{gathered}
$$

Here, $e_{\ell}, f_{\ell}$, and $g_{\ell}$ are respectively components of $\mathbf{E}, \mathbf{F}$, and $\mathbf{G}$, with $\ell=3,4, \ldots, 11$ as their index number. Note that because $e_{1}=K, e_{2}=H, e_{12}=e_{13}=e_{14}=0$ are constant for all $\lambda$ along a streamline, the upgrading of the numerical procedure needs to be done for $e_{3}, e_{4}, e_{5}, \ldots, e_{11}$ only. The Van Leer limiter function

$$
\phi(r)=\left\{\begin{array}{cc}
0 & r<0 \\
\frac{2 r}{r+1} & r>0
\end{array}\right.
$$

is used throughout this report, because there is no substantial difference in the numerical results between applying different limiter functions (ref. 12).

When the vector $\mathbf{E}$ is upgraded by the high-resolution TVD scheme, one can follow, in sequence, the same procedure as in the Godunov scheme. The TVD scheme leads to better accuracy in the continuous region and higher resolution across discontinuities, as shown in the section Test Problems. 


\section{SOLUTION OF THE PSEUDO-THREE-DIMENSIONAL RIEMANN PROBLEM}

As a building block, the Riemann problem with its solution play an important role in the Godunov-type schemes in the numerical solution of inviscid, compressible, perfect gas, flow problems. According to the Godunov scheme, a three-dimensional Riemann solver is required in our numerical procedure (fig. 2(a)). As previously mentioned, the exact solution to a general three-dimensional Riemann problem is not yet known. Even if it were available the procedure would be complicated and inefficient. Chang and Hsiao (ref. 9) offer a detailed description of the complexity of the higher dimensional Riemann problem. A practical remedy is to use dimensional splitting. The dimension-splitting technique reduces the number of initial constant states from four in the full three-dimensional Riemann problem to two in the so-called pseudo-three-dimensional Riemann problem (fig. 2(b)). This replaces the exact, but unavailable, three-dimensional Riemann problem solution with four approximate ones that are already known. In this section, we will study the pseudo-three-dimensional Riemann problem (PRP) and its solution.

Given two uniform states $\mathbf{Q}_{t}$ (top state) and $\mathbf{Q}_{b}$ (bottom state) in a three-dimensional space, we assume that the two states are separated by a plane and that the direction of the interaction line is known (refer to the preceding paragraph and figs. (2(b) and (c)). These conditions are satisfied in the aforementioned Godunov scheme. As in figure 3, we chose the direction of interaction line as the $z$-direction and the plane that separates $\mathbf{Q}_{t}$ and $\mathbf{Q}_{b}$ (separating plane) as the $y z$-coordinate plane. Thus, we have a Cartesian $x y z$-coordinate system. In this system, the Euler equations for three-dimensional steady flow have the form

$$
\begin{gathered}
\frac{\partial(\rho u)}{\partial x}+\frac{\partial(\rho v)}{\partial y}+\frac{\partial(\rho w)}{\partial z}=0 \\
u \frac{\partial u}{\partial x}+v \frac{\partial u}{\partial y}+w \frac{\partial u}{\partial z}+\frac{1}{\rho} \frac{\partial p}{\partial x}=0 \\
u \frac{\partial v}{\partial x}+v \frac{\partial v}{\partial y}+w \frac{\partial v}{\partial z}+\frac{1}{\rho} \frac{\partial p}{\partial y}=0 \\
u \frac{\partial w}{\partial x}+v \frac{\partial w}{\partial y}+w \frac{\partial w}{\partial z}+\frac{1}{\rho} \frac{\partial p}{\partial z}=0 \\
u \frac{\partial}{\partial x}\left(\frac{p}{\rho^{\gamma}}\right)+v \frac{\partial}{\partial y}\left(\frac{p}{\rho^{\gamma}}\right)+w \frac{\partial}{\partial z}\left(\frac{p}{\rho^{\gamma}}\right)=0
\end{gathered}
$$

where $u, v$, and $w$ are the velocity components in the new Cartesian coordinate system and $p$ and $\rho$ are pressure and density, respectively. The two given states can be written as

$$
\left\{\begin{array}{l}
Q_{t}=\left(u_{t}, v_{t}, w_{t}, p_{t}, \rho_{t}\right)^{T} \\
Q_{b}=\left(u_{b}, v_{b}, w_{b}, p_{b}, \rho_{b}\right)^{T}
\end{array}\right.
$$




$$
\frac{\partial}{\partial z}=0 \quad \text { and } \quad w=\text { constant }
$$

equation (25) is reduced to the Euler equations of two-dimensional steady flow:

$$
\begin{gathered}
\frac{\partial(\rho u)}{\partial x}+\frac{\partial(\rho v)}{\partial y}=0 \\
u \frac{\partial u}{\partial x}+v \frac{\partial u}{\partial y}+\frac{1}{\rho} \frac{\partial p}{\partial x}=0 \\
u \frac{\partial v}{\partial x}+v \frac{\partial v}{\partial y}+\frac{1}{\rho} \frac{\partial p}{\partial y}=0 \\
u \frac{\partial}{\partial x}\left(\frac{p}{\rho^{\gamma}}\right)+v \frac{\partial}{\partial y}\left(\frac{p}{\rho^{\gamma}}\right)=0
\end{gathered}
$$

Consider next the two-dimensional Riemann problem of equation (27) with the initial condition (fig. 3)

$$
\mathbf{Q}^{0}= \begin{cases}\mathbf{Q}_{t}^{0} & x>0 \\ \mathbf{Q}_{b}^{0} & x<0\end{cases}
$$

where $\mathbf{Q}^{0}=(u, v, p, \rho)^{T}, \mathbf{Q}_{t}^{0}=\left(u_{t}, v_{t}, p_{t}, \rho_{t}\right)^{T}$, and $\mathbf{Q}_{b}^{0}=\left(u_{b}, v_{b}, p_{b}, \rho_{b}\right)^{T}$. This is a standard two-dimensional Riemann problem and can be solved based on equation (27) and the Rankine-Hugoniot relations by using Newton's iteration. The solution, denoted by $\mathbf{Q}^{0}$, generally consists of all the elementary waves: the oblique shock (+), the slipline (0), and the Prandtl-Meyer (-) expansion. Based on $\mathbf{Q}^{0}$, the Riemann solution of equation $(25)$ is constructed in the following way:

$$
\mathbf{Q}= \begin{cases}\left(u, v, w_{t}, p, \rho\right)^{T} & x>x_{s} \\ \left(u, v, w_{b}, p, \rho\right)^{T} & x<x_{s}\end{cases}
$$

where $x_{s}$ is the $x$-coordinate of the slip-surface.

Since $\partial \mathbf{Q} / \partial z=0$ and $w_{t}$ and $w_{b}$ are given constants, the conditions of equation (26) are satisfied. Therefore, equation (29) is the solution of equation (26); thus, the name "pseudo-three-dimensional Riemann problem solution." Physically, $\mathbf{Q}$ can be regarded as translating in the $z$-direction along the upper part (above the 
slip-surface) and the lower part (below the slip-surface) of $\mathbf{Q}^{0}$ at different speeds $\left(w_{t}\right.$ and $\left.w_{b}\right)$ across the slipsurface. A numerical example will be illustrated in the section Test Problems.

If a solid boundary is present, only one initial state, say, $\mathbf{Q}_{t}$ is considered in the pseudo-three-dimensional Riemann problem (fig. 2(d)). The problem can then be termed a pseudo-three-dimensional boundary Riemann problem (PBRP). Similar to the preceding procedure, we first solve the corresponding two-dimensional boundary Riemann problem and obtain the solution $\mathbf{Q}^{0}=(u, v, p, \rho)^{T}$ and let $\mathbf{Q}=\left(u, v, w_{t}, p, \rho\right)^{T}$. $\mathbf{Q}$ is then the solution of the pseudo-boundary Riemann problem since equation (26) is satisfied.

In the rest of this section, for completeness, we briefly describe the solution of the two-dimensional Riemann problem and two-dimensional boundary Riemann problem. The procedure is the same as in reference 4 , which can provide more detail.

As an analogue to the one-dimensional Riemann problem for unsteady flow, the standard Riemann problem for two-dimensional steady flow is an initial value problem of the system shown in equation (27) with a discontinuous initial condition of equation (28):

$$
\mathbf{Q}=(u, v, p, \rho)^{T}= \begin{cases}\mathbf{Q}_{t} & x>0 \\ \mathbf{Q}_{b} & x<0\end{cases}
$$

where the subscripts $t$ and $b$ denote, respectively, top and bottom states (fig. 3(b)) and are counterparts to the left and right states in one-dimensional unsteady flow. For brevity, the superscript " 0 " has been dropped.

The solution of the two-dimensional Riemann problem is self-similar in the variable $y / x$ and consists of three types of elementary waves: the oblique shock (+), the Prandtl-Meyer expansion ( - ), and the slipline (0) (fig. 3).

Let $\mathbf{Q}_{0}=\left(u_{0}, v_{0}, p_{0}, \rho_{0}\right)^{T}$ and $\mathbf{Q}=(u, v, p, \rho)^{T}$ be the states across one of the,+- , and 0 elementary waves and $\alpha=p / p_{0}$. Then, through any state $\mathbf{Q}_{0}$, with $p$ as a parameter, there are two families of state connecting to $\mathbf{Q}_{0}$ : the compression states $\left(p \geq p_{0}, \alpha \geq 1\right)$ and the expansion states $\left(p \leq p_{0}, \alpha \leq 1\right)$. The two families join smoothly at $\mathbf{Q}_{0}$ and can be regarded as a single family. This makes it possible to apply Newton's iterative procedure in the solution of the Riemann problem. The central issue in the solution procedure is to find common values of $p$ and $\theta\left(p^{*}, \theta^{*}\right)$ at the slipline (fig. 4). The following details the solution:

(1) In the $p \theta$-plane (fig. 4), there are two curves passing through the two given states $\mathbf{Q}_{0}=\mathbf{Q}_{\mathbf{t}}$ and $\mathbf{Q}_{0}=\mathbf{Q}_{b}$. They are, respectively, defined as

$$
\theta=\Phi_{t}(p)= \begin{cases}\theta_{t}+\tan ^{-1}\left[\frac{\alpha-1}{\gamma M_{t}^{2}-\alpha+1}\left(\frac{2 \gamma M_{t}^{2}}{(\gamma+1) \alpha+\gamma-1}-1\right)^{1 / 2}\right] & p \geq p_{t} \\ \theta_{t}+v\left(M_{t}\right)-v(M) & p \leq p_{t}\end{cases}
$$


and

$$
\theta=\Phi_{b}(p)= \begin{cases}\theta_{b}-\tan ^{-1}\left[\frac{\alpha-1}{\gamma M_{b}^{2}-\alpha+1}\left(\frac{2 \gamma M_{b}^{2}}{(\gamma+1) \alpha+\gamma-1}-1\right)^{1 / 2}\right] & p \geq p_{b} \\ \theta_{b}-v\left(M_{t}\right)+v(M) & p \leq p_{b}\end{cases}
$$

where $v(M)$ is the well-known Prandtl-Meyer function. These curves are shown in figure 4.

(2) The Newton iterative procedure is then employed to find the intersect $\left(p^{*}, \theta^{*}\right)$ of the two curves. The object function to be driven to zero in the Newton procedure is

$$
f(p)=\Phi_{t}(p)-\Phi_{b}(p)
$$

and the intersect of the tangent lines passing through $\mathbf{Q}_{t}$ and $\mathbf{Q}_{b}$ is used as an initial guess to the solution. In practice, we use numerical derivatives to replace the analytical ones. Usually it takes 2 to 4 iterations to converge to a tolerance of $\varepsilon \leq 10^{-6}$.

(3) With the slipline values $\left(p^{*}, \theta^{*}\right)$ calculated, we evaluate the new $\rho$ on either side across the slipline by the Hugoniot relation,

$$
\rho= \begin{cases}\rho_{0}\left[\frac{(\gamma+1) \alpha+\gamma-1}{(\gamma-1) \alpha+\gamma+1}\right] & \alpha \geq 1 \\ \rho_{0} \alpha^{1 / \gamma} & \alpha<1\end{cases}
$$

then the speed $q$ with the total enthalpy $H$ of the corresponding cell. Finally, the velocity components are available by

$$
u=q \cos \theta^{*} \quad \text { and } \quad v=q \sin \theta^{*}
$$

These newly calculated flow variables $u, v, p^{*}$, and $\rho$ represent the state at the slipline. Recall that in our Lagrangian formulation, a slip-surface coincides with the cell interface (a stream surface); these data are used to calculate cell interface fluxes as described in the previous section.

At the solid boundary, the flow inclination condition is imposed and one of the curves in the $p \theta$-plane, say, $\theta=\Phi_{b}(p)$ degenerates to a straight line $\theta=\theta^{*}=$ constant parallel to the $p$-axis (fig. 4$)$. In reference 4 , this particular problem is termed "boundary Riemann problem." The solution of a boundary Riemann problem is similar to the aforementioned procedure but a different object function is used:

$$
f(p)=\Phi_{t}(p)-\theta^{*} \quad \text { or } \quad f(p)+\Phi_{b}(p)-\theta^{*}
$$


At this stage, because of the application of Riemann solution and the Godunov scheme to the new Lagrangian formulation, the slip surface (contact discontinuity) resolution always remains sharp.

Consider a typical case in which a slip surface exists between two continuous flows and coincides with the cell interfaces between cells $(i+1, j)$ and $(i, j), j=1,2, \ldots, n$. Thus,

$$
p_{i+1, j}^{k}=p_{i, j}^{k}
$$

and both $\mathbf{V}_{i+1, j}^{k}$ and $\mathbf{V}_{i, j}^{k}$ are parallel to the cell interfaces, whereas there are jumps in $\rho$ and the flow speed $q=|\mathbf{V}|$. In the marching, after interaction of the two flows, since the new cell interface follows exactly the new slip surface (a stream surface), these relations still hold at the new cell interface. Then the cell interface flux $\mathbf{F}_{i+1 / 2, j}^{k+1 / 2}$ on either side of the slip surface is continuous on the same side where it is derived, and still produces continuous solutions through the Godunov scheme along each side of the slip surface. The pressure is continuous in the whole region since it is continuous across the slip surface. However, the original discontinuities in $\rho$ and $q$ across the slip surface will remain because the marching evolution on each side of the slip surface is continuous. In other words, the slip surface remains sharp during the marching. In a special case in which both continuous flows are uniform, the flows remain unchanged during marching-forward as seen from the Godunov scheme.

When the slip surface does not coincide with a cell interface, for example, when it is generated by shock interaction, the cell through which the true slip surface passes will be considered an intermediate cell in the captured slip surface. Based on this argument, no more intermediate cells will be generated around the true slip surface; that is, no further smearing will occur during the marching. Moreover, application of a high-resolution TVD scheme (such as Sweby's) will help to reduce the number of the intermediate cells and render a sharper captured slip surface.

In the new three-dimensional Lagrangian approach, the resolution of slip surface is similar to its twodimensional counterpart, as has been described by Hui and Loh (ref. 12). Compared with the ever-smearing and deteriorating resolution of contact discontinuity in the Eulerian formulation, the new Lagrangian approach provides an excellent means to attack the problem of slip surface resolution.

\section{WELL-POSEDNESS OF THE CAUCHY PROBLEM IN GAS DYNAMICS AND ITS NUMERICAL TREATMENT}

For a supersonic flow of Mach number $\mathbf{M}>1$ everywhere, the Euler equations of gas dynamics, either in Eulerian or Lagrangian formulation, are of hyperbolic type. The numerical solution marches forward along a time-like direction, say, the $x$-direction in the Eulerian formulation or the flow direction $(\tau$ or $\lambda)$ in the new Lagrangian formulation. In any case, the well-known CFL condition rules the maximum marching step size order to maintain stability.

Another important factor that controls the numerical stability is the well-posedness of the Cauchy problem (initial problem) of the Euler equations. Because the well-posedness can be seen as a general CFL condition, if it is violated, the numerical procedure will eventually blow up. The property of the new Lagrangian system (13) in which the physical flow is closely followed makes the well-posedness analysis intuitive and straightforward. Consider a computational cell (a fluid particle) in figure 5, where the initial data are given on the time (distance) surface $\Gamma$. For the well-posedness of this local Cauchy problem of (13), the basic theory of hyperbolic equation (see Courant and Hilbert (ref. 13)) stipulates that $\Gamma$ must lie upstream of the domains of 
influence (i.e., the Mach cones) issuing from every point of $\Gamma$; in particular, those Mach cones issuing from the vertices of $\Gamma$.

When a supersonic flow passes through a strong shock, similar to the two-dimensional case (ref. 12) the Mach number drops and the Mach cones downstream of the shock expand to wider angles, while $\Gamma$ changes its inclination as well. (This is more pronounced in the $\tau$-variable form.) It is possible that at some marching step, the surface $\Gamma$ extends into the interior of the Mach cone (fig. 5(b)) and renders the local Cauchy problem illposed. Following the experience from the two-dimensional version (ref. 12), a remedy is to redirect the inclination (direction of normal vector) of the initial time (distance) surface of each cell so that even after the flow passes through the shock, the time surface still lies upstream of the Mach cones. Because of the flexibility of time (distance) surface, the redirection procedure can be performed not only at the initiation stage but also in the midst of computation without disturbing the formulation and any data already computed.

The same difficulty of well-posedness exists in the Eulerian formulation. However, as a result of the fixed time-like marching direction, say, the $x$-direction, one has to artificially rotate the reference frame and perform all the pertinent coordinate transformations. Marconi and Moretti (ref. 14) have used this approach. They employed a local coordinate rotation to their nonconservative, implicit shock-fitting scheme to assure the proper domain of dependence of the grid points; thus, supersonic velocity in the marching direction is maintained. For a conservative shock-capturing scheme, the procedure is expected to be even more cumbersome. In this regard, the Lagrangian formulation, by simply redirecting the time (distance) surface, is much easier than the Eulerian.

In addition, as in the two-dimensional case (ref. 12), note another advantage of the new Lagrangian formulation over its Eulerian counterpart: the scheme may march forward with a larger Courant number. This situation is illustrated in figure 5(d), assuming the initial surface coincides with the $x y$-plane. Because of the complexity of three-dimensional geometry, we present only a section of the entire configuration. Nevertheless, the Lagrangian formulation appears to have an "optimal" Courant number since its marching direction (the flow direction) is right in the middle of the Mach cones; whereas the maximum Eulerian marching step in the fixed $x$-direction does not exceed the intersection of the Eulerian cell interface with any Mach wave.

\section{TEST PROBLEMS}

To test the accuracy and robustness of the new three-dimensional Lagrangian method, we applied it to several test examples and compared the results to the exact solutions, existing numerical solutions, or experimental results (when available).

The first example is a pseudo-three-dimensional Riemann problem. Two flows with states $\mathbf{Q}_{1}$ and $\mathbf{Q}_{2}$ as described in figure 6 are separated by the separating plane and begin to interact with each other at the interacting line (fig. 6(a)). The problem is analyzed in the section Solution of the Pseudo-Three-Dimensional Riemann Problem and the exact solution can be easily obtained by using a standard two-dimensional Riemann solver. In figure 6, parts (b) and (c), we illustrate the pressure and density distributions along a time surface in the $x y$-plane (interaction plane). Both numerical results by the Godunov and TVD schemes agree well with the exact solution. In particular, the slipline resolution is so crisp that there is practically no point in between. The TVD scheme produces more accurate results in the continuous part of the flow and a sharper profile across the shock. In this example, a mesh of $100 \times 50$ cells (in the $y z$-plane) and the $\tau$ conservation form (10) are employed.

In the second example, we consider a truly three-dimensional, initial value-boundary value problem-the supersonic inviscid corner flow. The geometrical configuration is shown in figure 7(a). Two intersecting wedges, both with angles of $9.5^{\circ}$, form an axial corner over which there is a Mach 3 flow. The flow field consists of two planar wedge shocks, two embedded shocks, a corner shock, and the shear layers (slip-surfaces) as shown in 
figure 7. West and Korkegi (ref. 15) carried out an experiment for this case, which we will use as a comparison with our numerical results. In their experiment, the turbulent boundary layers are thin and the effect on the shock displacement is minimal. Thus, the overall picture can be considered an inviscid phenomenon, except near the walls. We employ a mesh of $45 \times 45$ points in the $y z$-plane for the computation. We used the threedimensional color-displaying package FAST developed by NASA Ames Research Center for presenting the numerical results. The color figures are presented here in black and white.

Figure 7(b) illustrates the $u$-velocity contours on a typical $\lambda$-plane. The corner shock, embedded shocks, and two-dimensional wedge shocks are clearly shown. In particular, the triangular slip-surfaces are distinct and sharp. They all agree well with the experimental locations determined by West and Korkegi (ref. 15). In figure $7(\mathrm{c})$, we present the isobars on the same $\lambda$-plane. All the shocks are still clearly visible. The slip-surfaces disappear because the pressure across them is continuous. However, a gentle decrease in pressure from the corner shock toward the walls and the wall corner appears. Figure 7(c) shows the isopycnics (density contours) on the same $\lambda$-plane. The density peaks at the two triple points, where the embedded shock, the wedge shock, and the corner shock meet one another. Figure 7(e) displays how the grid on a typical plane deforms with the flow; the grid remains straight until the shock is encountered and changes to conform with the deflection of the streamline. The shock angle agrees well with the exact solution for the two-dimensional wedge shock. Figure 7(f) shows results by Liou and Hsu (ref. 16), which are based on solving the Navier-Stokes equations with a very high Reynolds number in the standard Eulerian formulation. A similar mesh of $45 \times 45$, but nonuniform, grid points with finer size at the walls are used in their example. Note that their slip-surfaces are less clear.

In the third problem, we calculate a Mach 4 flow past a delta wing with a $10^{\circ}$ angle of attack. In this case, we intend to show the robustness of our method in handling different body shapes in supersonic flow. The symmetrical wing body is illustrated in figure 8 (a). The semispan angle $\delta=40^{\circ}$, the semithickness angle $\phi=2.5^{\circ}$, and its longitudinal cross section is diamond shaped. Initially, the vector $\mathbf{T}$ is chosen to be the unit vector along the projection of wing edge $\mathrm{AC}$ on $y z$-plane. We use the $\lambda$ conservation form and a $50 \times 50$ mesh is employed in the computation. Figure 8(b) illustrates the isobars at different stations around the wing body; the bow shock at the top, the expansion fan at the bottom and other expansion fans arising from the central ridge, and the trailing edge are clearly displayed. Figure 8(c) shows the isobars on the upper wing body surface (a stream surface), the shock at the leading edge, and the expansions at the central ridge and the trailing edge. The little "zigzags" in the contours are due to the nonalignment of the vector $\mathbf{T}$ with the leading edge or ridge line in each cell. $\mathbf{T}$ was chosen for numerical stability (see Solution of the Pseudo-Three-Dimensional Riemann Problem), and the unpleasant "zigzags" soon disappear on other stream surfaces. Figure 8, parts (d) and (e) show the isobars on two typical stream surfaces parallel to, but above and below, the wing body. In figure 8, parts (f) and (g), we demonstrate the isobars and isopycnics on the symmetric plane. Bow shock, trailing shock, three expansion fans and, in particular, the slipline are clearly displayed. Evident in figure $8(\mathrm{~g})$, is a very weak wave as a result of the reflection of the ridge fan from the leading-edge shock. Furthermore, in figure 8 , parts (h) and (i), we present the detailed pressure and density distribution along a typical time line on the symmetric plane to show the quality of the solution; in particular, a clear discontinuity across the slipline (surface) is shown in figure 8(i). Finally, figure 8 , parts $(\mathrm{j})$ and $(\mathrm{k})$ show the isobars and isopycnics on a typical spanwise cross section parallel to the symmetric plane. They bear a similarity to those in the symmetric plane.

In the last example, we compute a novel three-dimensional Riemann problem. For many years, the threedimensional Riemann problem has been a topic of constant interest and has remained unsolved. To our knowledge, we are the first to calculate the numerical solution for a problem of this type. The capability of our scheme is based on its excellent behavior across the slip-surface. Having no existing solution for comparison, we use grid meshes of $50 \times 50$ and $100 \times 100$ and confirm that the results are similar. The Riemann problem in question is shown in figure 9(a) where the initial conditions are given in the four quadrants of the $y z$-plane. For better understanding of the flow, we choose identical states in the first and third, and second and fourth quadrants; that is, 


$$
\begin{gathered}
\mathbf{Q}_{1}=\left(u_{1}, v_{1}, w_{1}, p_{1}, \rho_{1}\right)^{T}+(5,0,0,0.25,0.5)^{T} \\
\mathbf{Q}_{2}=\left(u_{2}, v_{2}, w_{2}, p_{2}, \rho_{2}\right)^{T}=(3.5,0,0,1,1)^{T}
\end{gathered}
$$

Figure 9 (b) shows the isobars on a typical $\lambda$ plane where the flow is fully developed. The compression, expansion kernels, two-dimensional shocks, and expansion fan regions are clearly observed. Similar to the twodimensional case, between a shock and its corresponding region, there exists a region of uniform pressure. The density contours are presented in figure 9 (c). The slip-surfaces are crisp because they are never smeared by the Lagrangian numerical method. Figure 9(d) demonstrates the contours of $u$-velocity component. In figure 9 , parts (c) and (d), the slip-surfaces form a pair of curved symmetrical surfaces. In all parts of figure 9, the compression kernel consists of a singular point that is formed by the collision of two intersecting, two-dimensional shocks and the quick expansions around it. This simple flow structure strongly contrasts to the structure in the corner flow (fig. 7). Although formed by two intersecting wedge shocks, the latter is much more complicated as a result of the solid walls. Figure 9 (e) shows a side view of the solution at one of the outermost stream surfaces. On this stream surface, the flow is identical to a two-dimensional Riemann problem solution. Figure 9(f) displays the shape of a slip-surface and several typical meshes. Note that the meshes are automatically deformed, expanded, or condensed according to the flow. Generally, our result agrees well with the qualitative description in the paper by Chang and Hsiao (ref. 9).

\section{CONCLUDING REMARKS}

In this report, we have developed a new Lagrangian method for steady three-dimensional supersonic flow computation. The method employs Lagrangian time $\tau$ (or Lagrangian distance $\lambda$ ) and the stream functions as the independent variables in place of the Cartesian coordinates $x, y$, and $z$. The boundary conditions are satisfied exactly and the method is robust, accurate, and particularly appropriate for complex geometry. The new approach is superior to the conventional Lagrangian (or Lagrangian-Eulerian) approach because the geometrical quantities are handled by conservation laws (compatibility equations). Unlike any Eulerian method, the new Lagrangian approach is characterized by the following features:

1. A computational cell is literally a fluid particle and flow physics is closely followed. The computation is truly multidimensional rather than the usual one-dimensional splitting.

2. A slip-surface is always crisply resolved without any detection and special treatment since a computational cell remains identical to the same fluid particle all the time.

3. Grid is automatically generated along the stream tubes as part of the solution. No a priori grid generation is needed.

4. The inherent parallelism of the Lagrangian approach, that each computational cell can be considered a fluid particle and a single processor interacting with its surrounding neighbors, lends itself to simple implementation of massively parallel computation on machines such as the CM-2 to CM-5. (This has been confirmed by the computations of two-dimensional version (ref. 17).)

In summary, the Lagrangian approach resolves both discontinuities (shocks and slip-surfaces) sharply in complicated geometry; once combined into massively parallel computation, we believe it will become a powerful tool for steady supersonic flow computation. Research on the Lagrangian approach to transonic and subsonic inviscid compressible flows is currently underway. 


\section{REFERENCES}

1. Roe, P.L.: A Survey of Upwind Differencing Techniques. International Conference on Numerical Methods in Fluid Dynamics, 11 th, (Lecture Notes in Physics, Vol. 323), D.L. Dwoyer, et al., eds., Springer, New York, 1989, pp. 69-78.

2. Belytschko, T.; and Hughes, T.J.R., eds.: Computational Methods for Transient Analysis, Ch. 8, NorthHolland, Amsterdam, 1983.

3. Harten, A.: ENO Schemes with Subcell Resolution. J. Comput. Phys., vol. 83, no. 1, 1989, pp. 148-184.

4. Loh, C.; and Hui, W.H.: A New Lagrangian Method for Steady Supersonic Flow Computation. Pt. 1: Godunov Scheme. J. Comput. Phys., vol. 89, no. 1, 1990, pp. 207-240.

5. Hui, W.H.; and Van Roessel, H.: Unsteady Three-Dimensional Flow Theory via Material Functions. Unsteady Aerodynamics—Fundamentals and Applications to Aircraft Dynamics, AGARD CP-386, 1985, S1.

6. Loh, C.Y.; and Hui, W.H.: Application of Lagrangian Time to Steady Supersonic Airfoil. AIAA Paper 89-1963, 1989.

7. Sweby, P.K.: High-Resolution Schemes Using Flux Limiters for Hyperbolic Conservation Laws. SIAM J. Numer. Anal., vol. 21, no. 5, 1984, pp. 995-1011.

8. Godunov, S.K.; Difference Method of Numerical Computations of Discontinuous Solutions in Hydrodynamic Equations. Matem. Sbornik, vol. 47, 1959, pp. 271-301.

9. Chang, T.; and Hsiao, L.: The Riemann Problem and Interaction of Waves in Gas Dynamics. Wiley, New York, 1989.

10. Harten, A.; Hyman, J.M.; and Lasc, P.D.: Finite Difference Approximations and Entropy Conditions for Shocks. Commun. Pure Appl. Math., vol. 29, no. 3, 1976, pp. 297-322.

11. Grandall, M.G.; and Majda, A.: Monotone Difference Approximations for Scalar Conservation Laws. Math. Comput., vol. 34, Jan. 1989, pp. 1-21.

12. Hui, W.H.; and Loh, C.Y.: A New Lagrangian Method for Steady Supersonic Flow Computation. Pt. II: Slip-Line Resolution. Pt. III: Strong Shocks. J. Comput. Phys., vol. 103, no. 2, Dec. 1992, pp. 450-471.

13. Courant, R.; and Hilbert, D.: Methods of Mathematical Physics. Academic Press, New York, 1962.

14. Marconi, F.; and Moretti, G.: Three Dimensional Supersonic Flows with Subsonic Axial Mach Numbers. AIAA Paper 76-383, 1976.

15. West, J.E.; and Korkegi, R.M.: Supersonic Interaction in the Corner of Intersecting Wedges at High Reynolds Numbers. AIAA J., vol. 10, May 1972, pp. 652-656.

16. Liou, M.S.; and Hsu, A.T.: A Time Accurate Finite Volume High Resolution Scheme for Three Dimensional Navier-Stokes Equations. AIAA Paper 89-1994, 1989.

17. Liou, M.-F.; and Loh, C.Y.: Parallel Computing Using a Lagrangian Formulation. NASA TM-104446, 1991. 


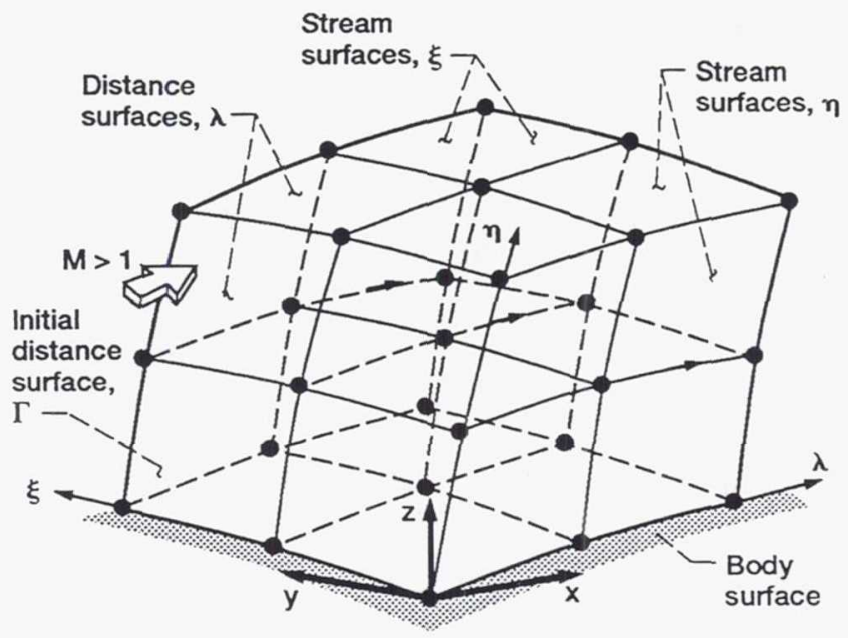

(a) Physical space.

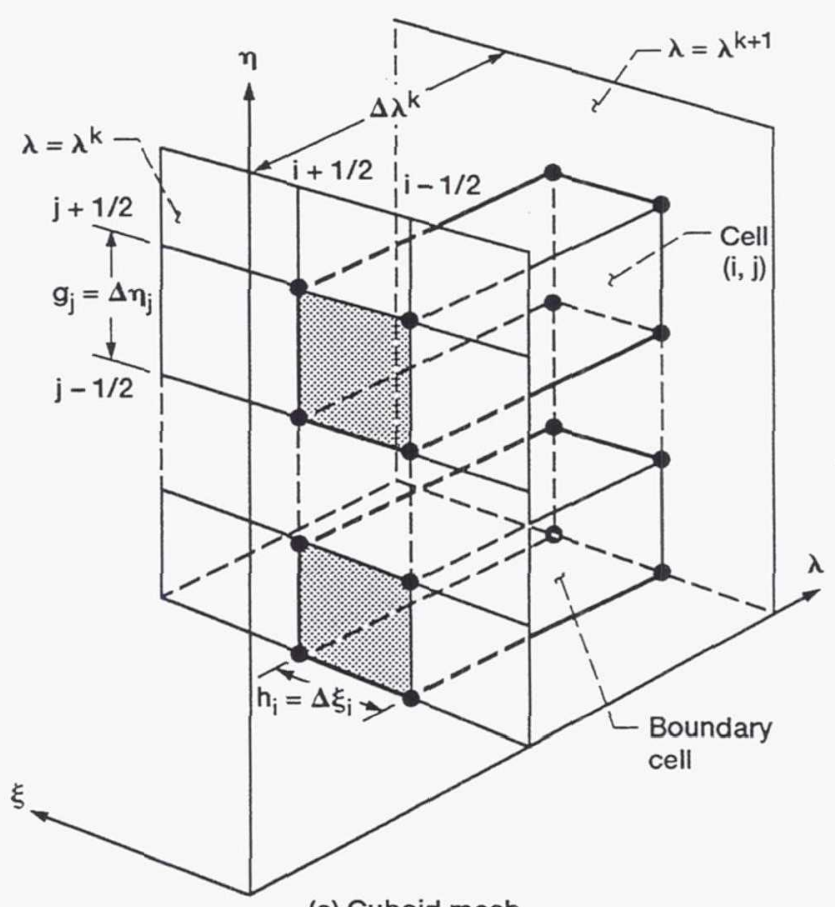

(c) Cuboid mesh.

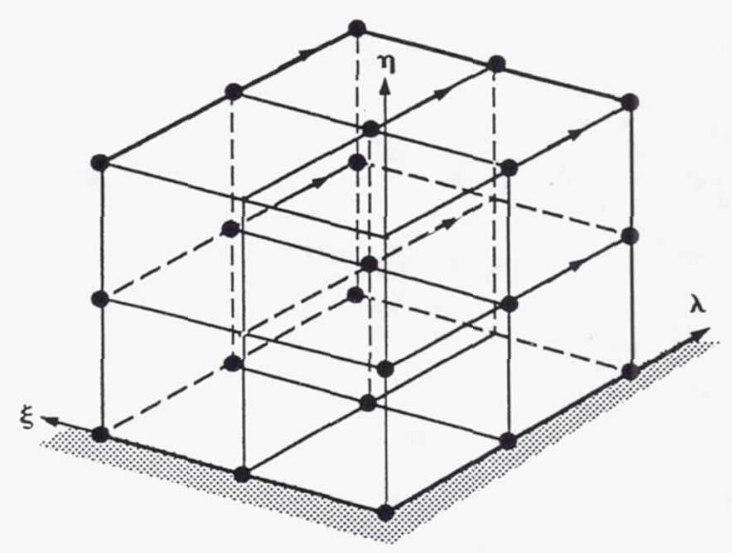

(b) Computational space.

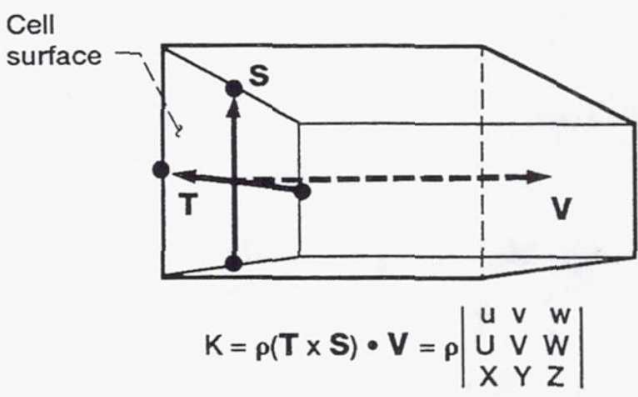

(d) Mass flux.

Figure 1.-Computational space and mesh. 


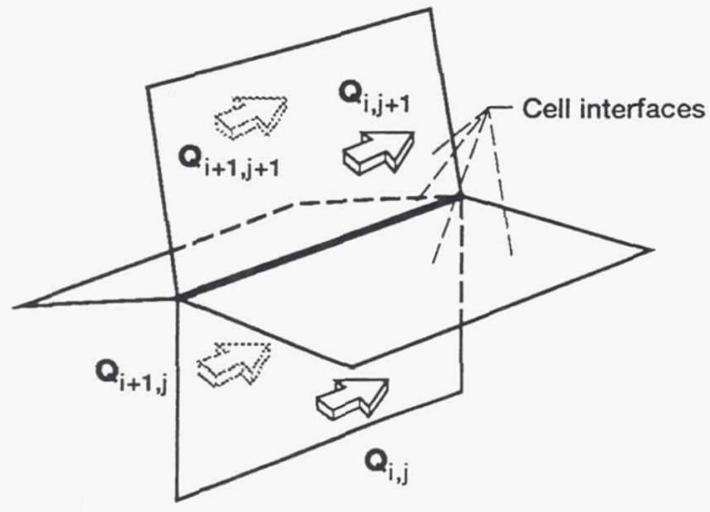

(a) Typical 3-D Reinmann problem in the Godunov scheme.

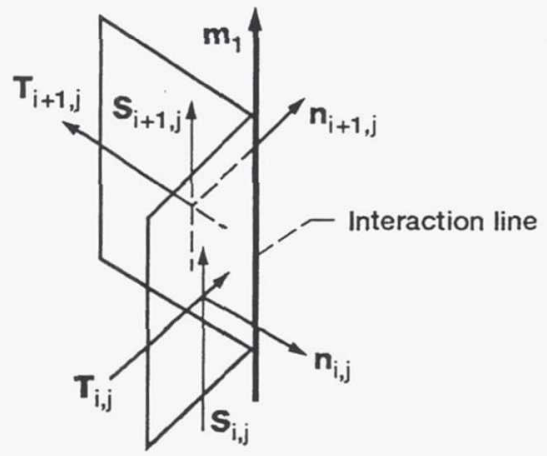

(c) Interaction line direction for pseudo-3-D Reinmann problem.

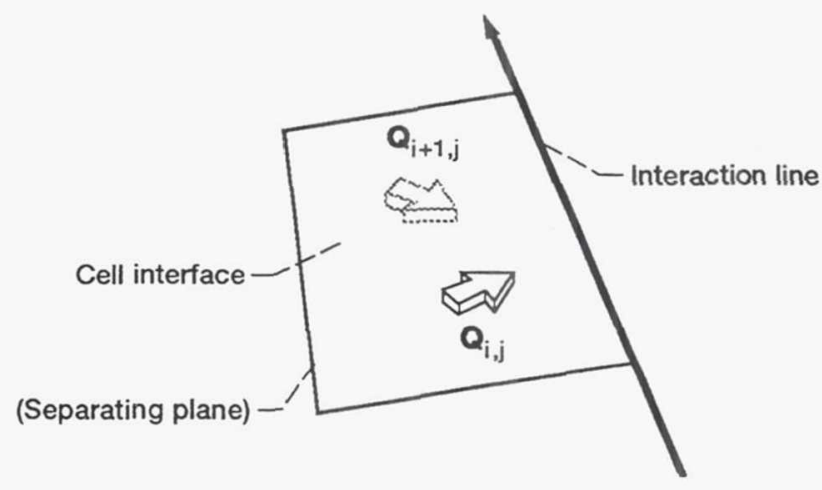

(b) Pseudo-3-D Reinmann problem with initial states $\mathbf{Q}_{i, j}$ and $\mathbf{Q}_{i+1, j}$

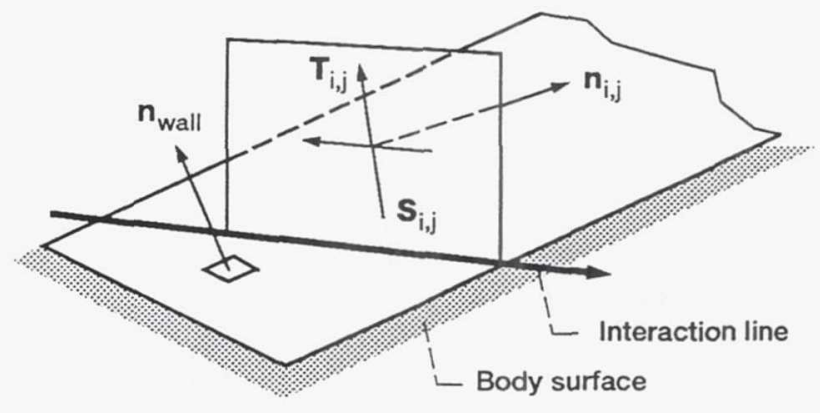

(d) Interaction line direction for pseudo-3-D boundary Reinmann problem.

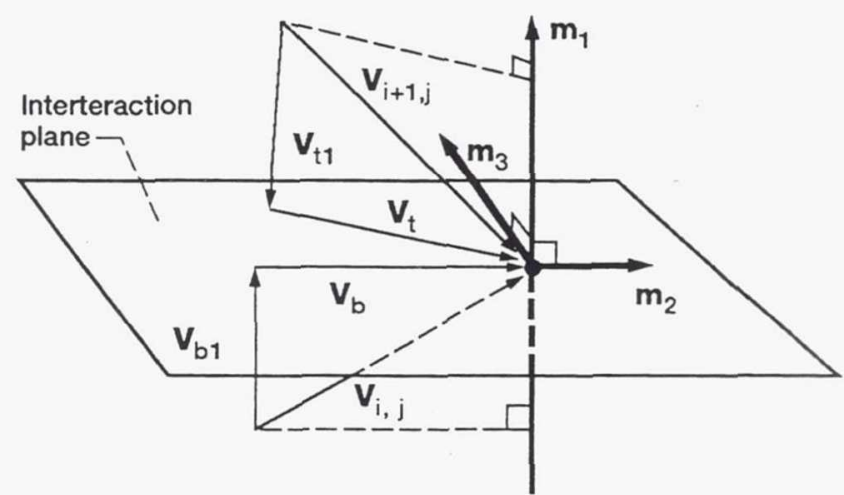

(e) Initial data for standard 2-D Reinmann problem on the interacting plane.

Figure 2-Determination of an interaction line. 


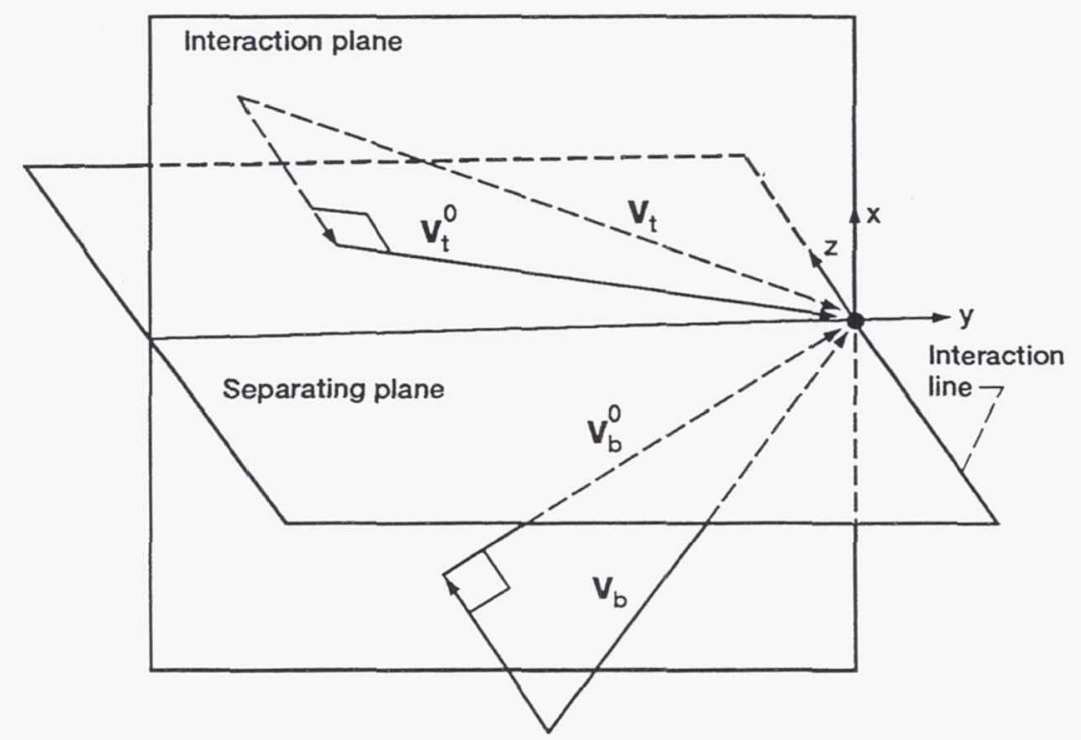

(a) Reduction of pseudo-3-D Riemann problem to 2-D Riemann problem.

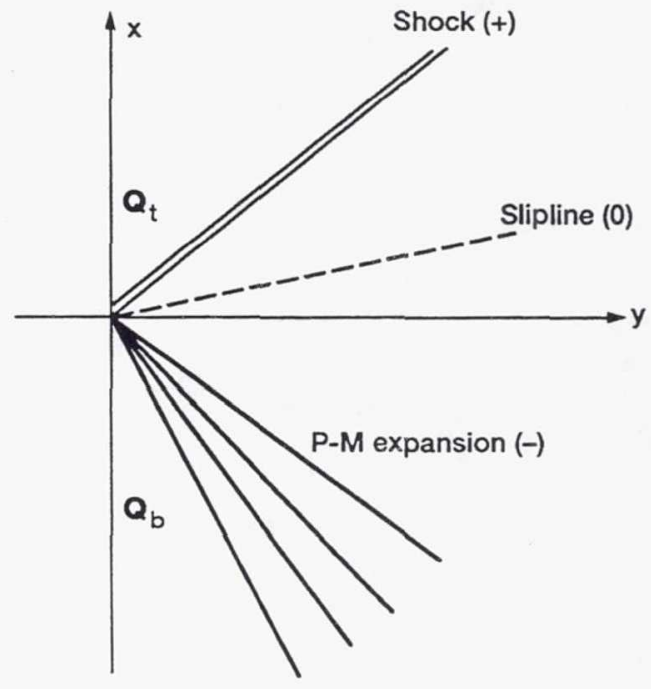

(b) The 2-D Riemann problem.

Figure 3.-The pseudo-3-D Riemann problem.

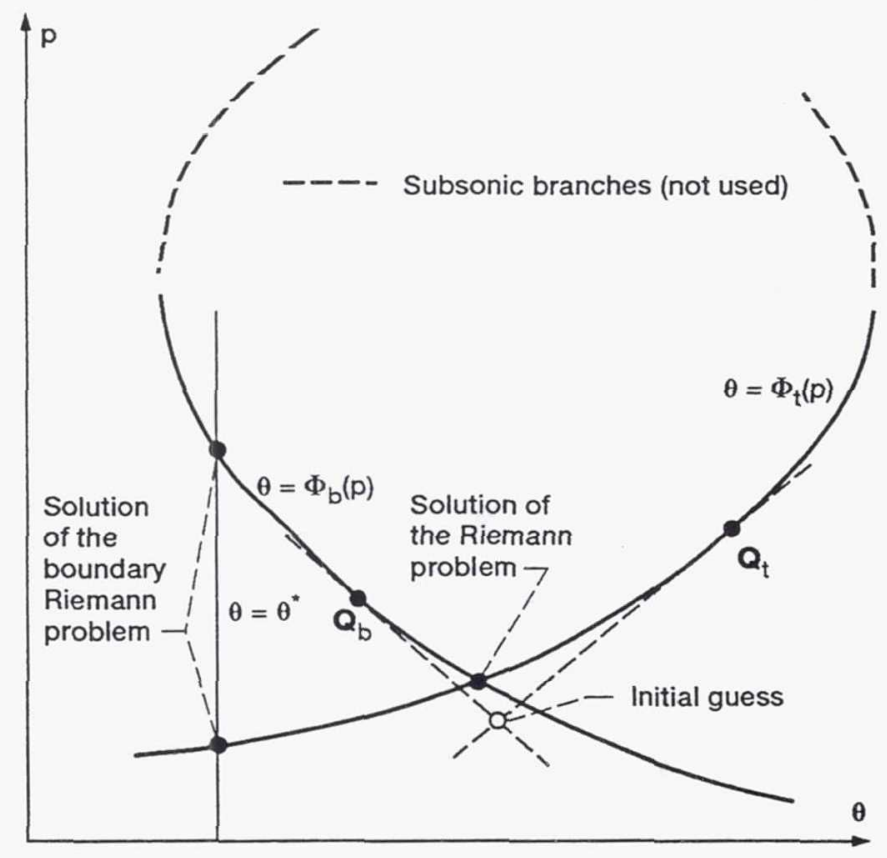

Figure 4.-Solution of 2-D Riemann problem. 


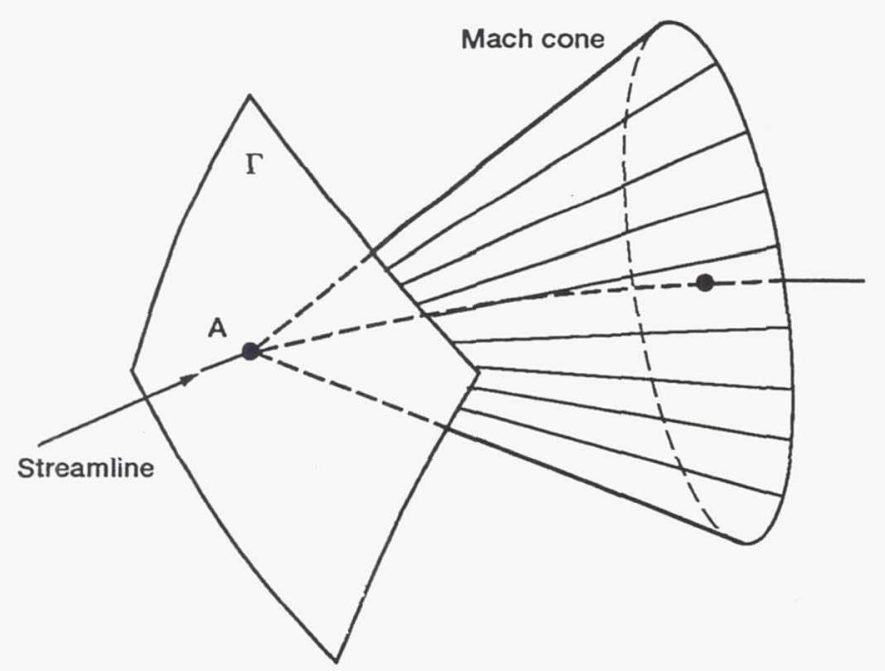

(a) $\Gamma$ lies upstream of Mach cone.

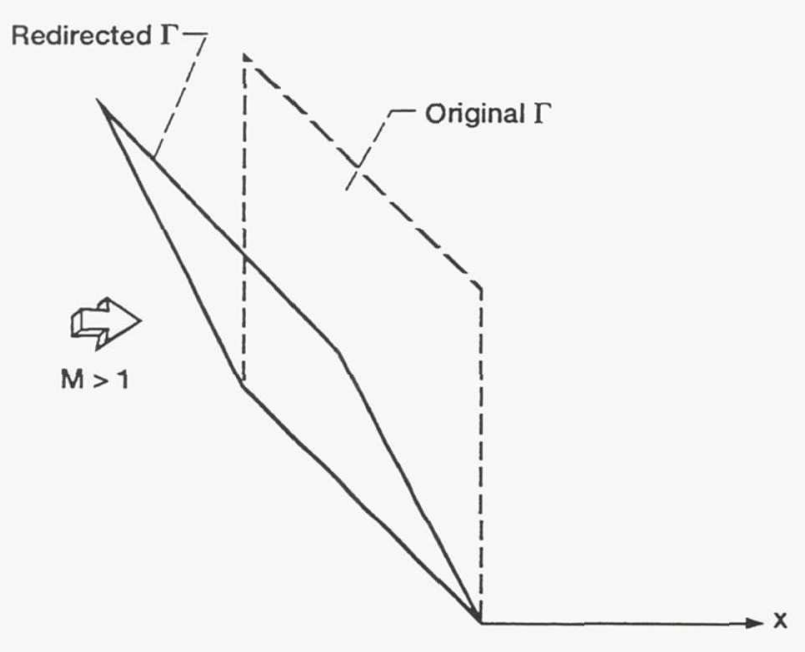

(c) Example of $\Gamma$ redirection.

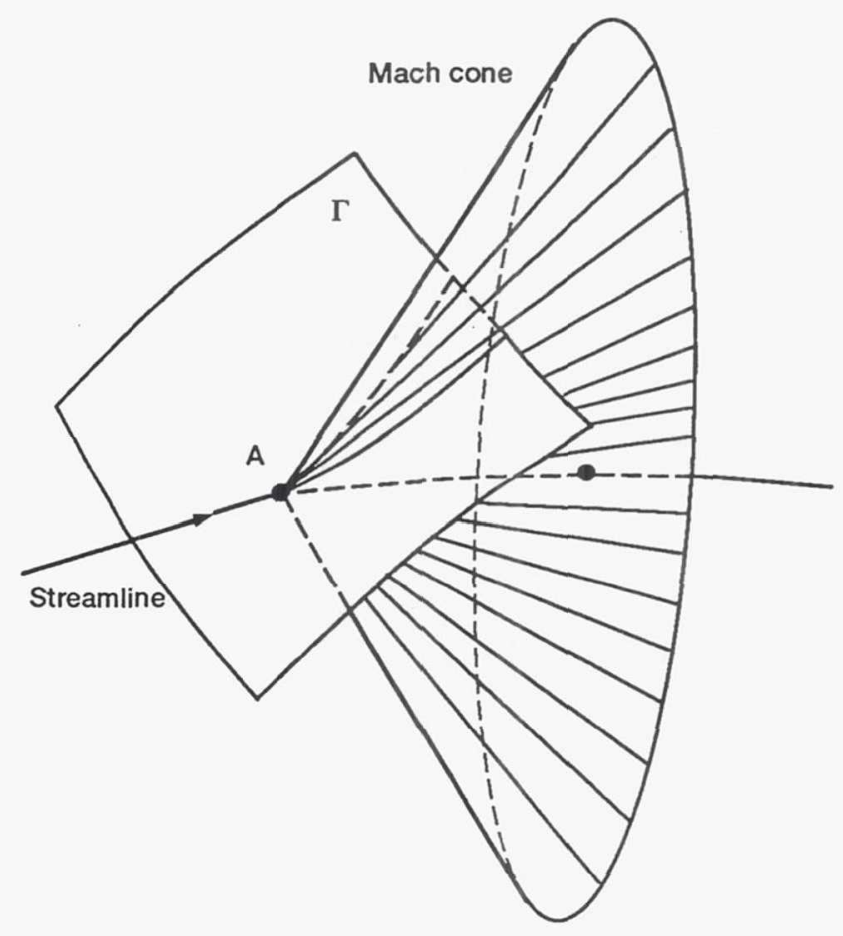

(b) $\Gamma$ turns into Mach cone.

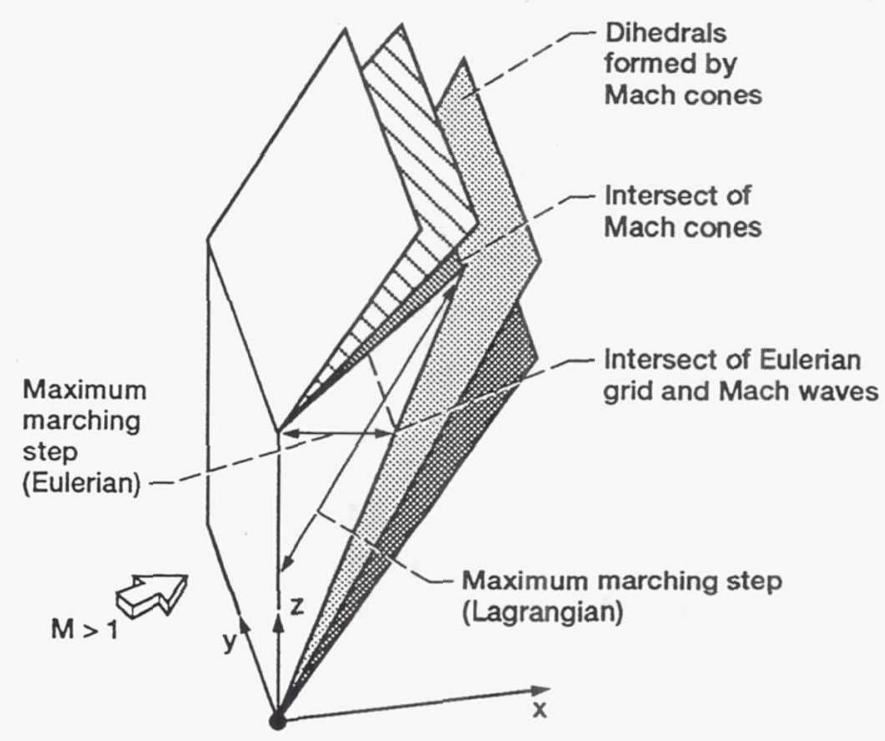

(d) Lagrangian Courant number (larger than Eulerian number).

Figure 5.-Well-posedness of the Cauchy problem. 


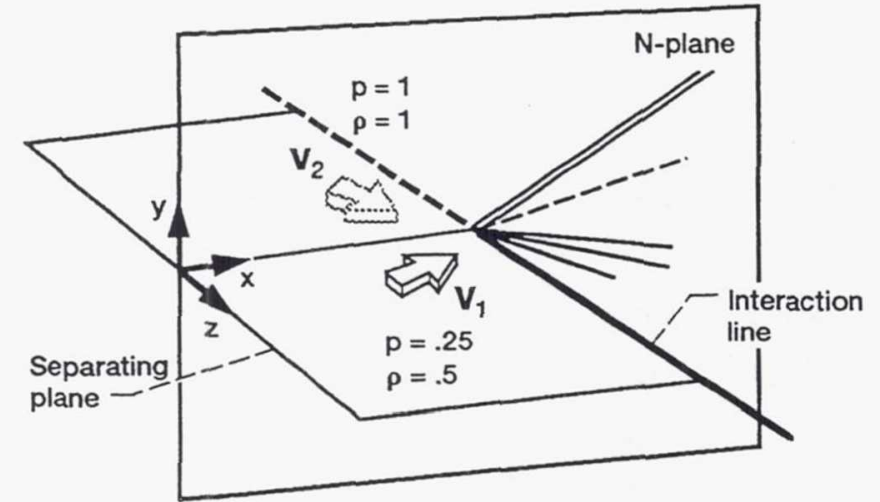

(a) Numerical solutions in $\mathrm{N}$-plane. $\left(\mathbf{V}_{1}=(3.3466,0,-1)\right.$ for $\mathrm{y}>0$, $\mathbf{V}_{2}=(2.8397,0,0.8)$ for $\left.\mathrm{y}<0.\right)$

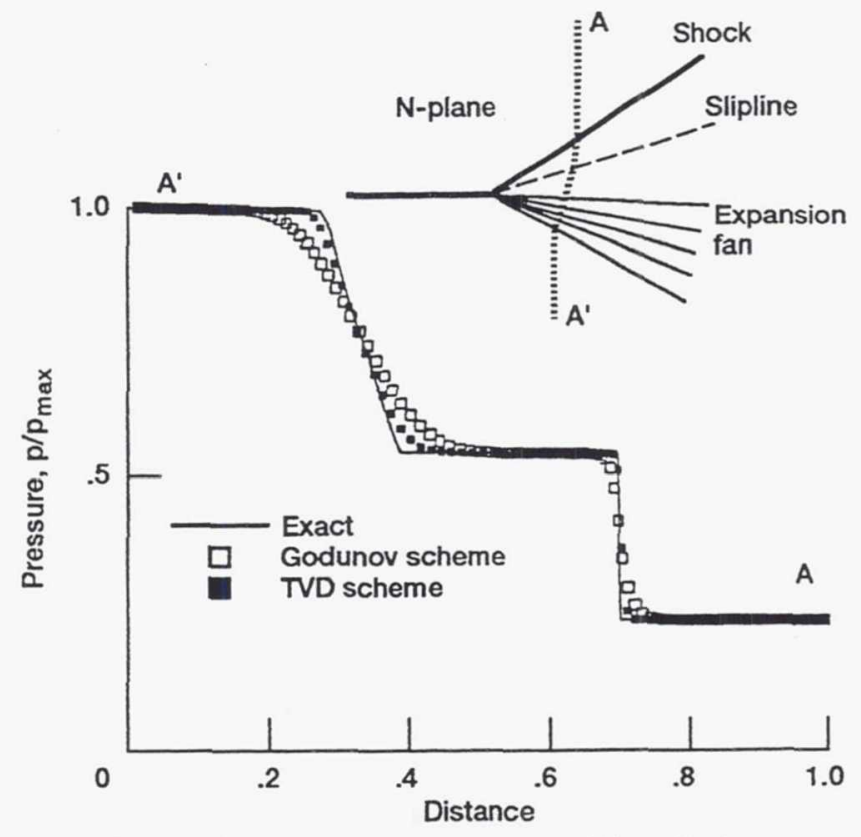

(b) Pressure distribution along time line $\mathrm{AA}^{\prime}$ on $\mathrm{N}$-plane.

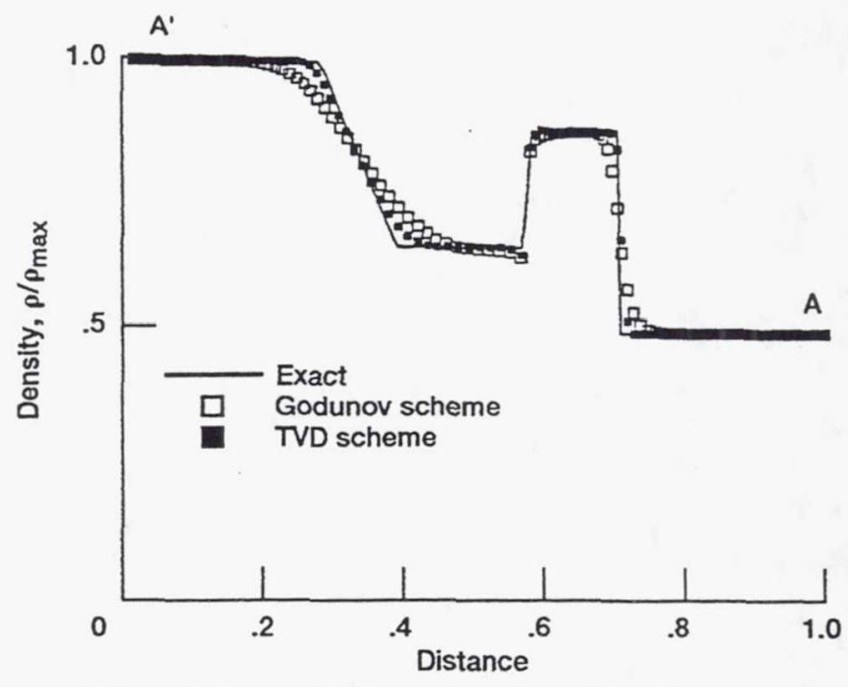

(c) Density distribution along time line $A A^{\prime}$ on $\mathrm{N}$-plane.

Figure 6.-A pseudo-3-D Riemann problem. 


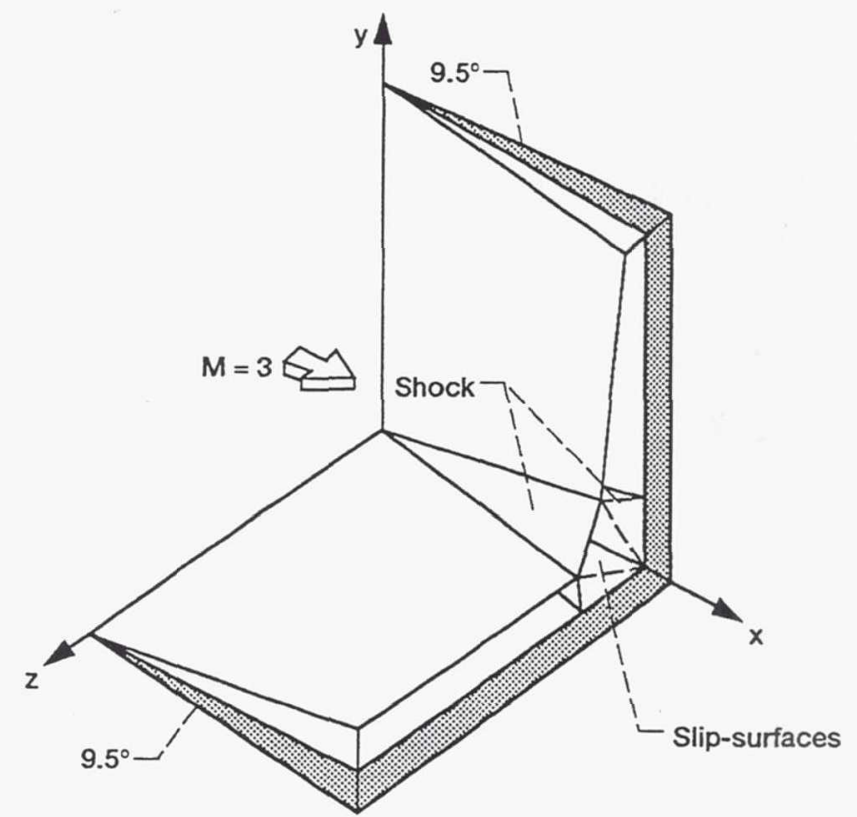

(a) Sketch of the problem.

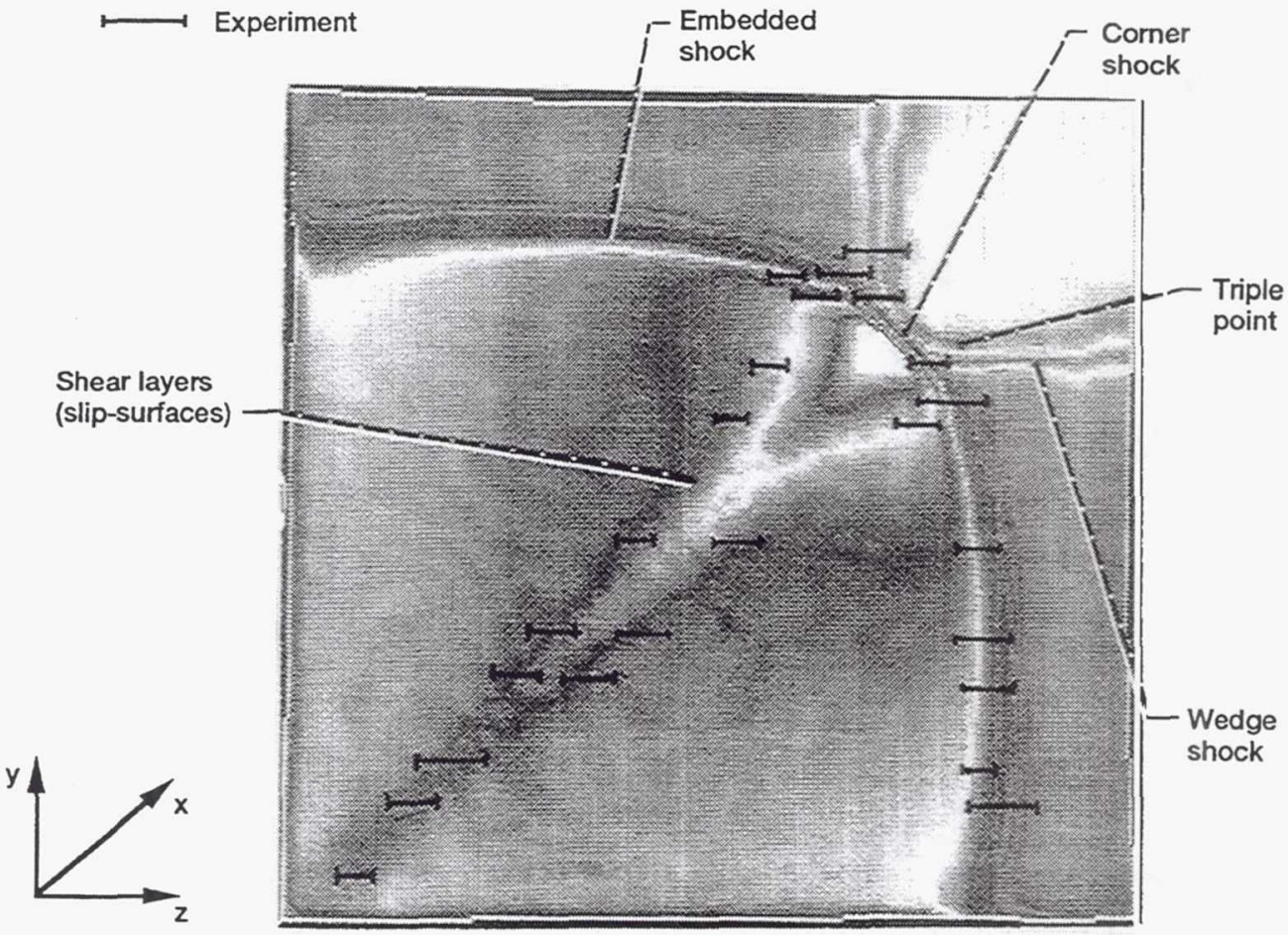

(b) u-velocity contours.

Figure 7.-Supersonic flow past a corner. 


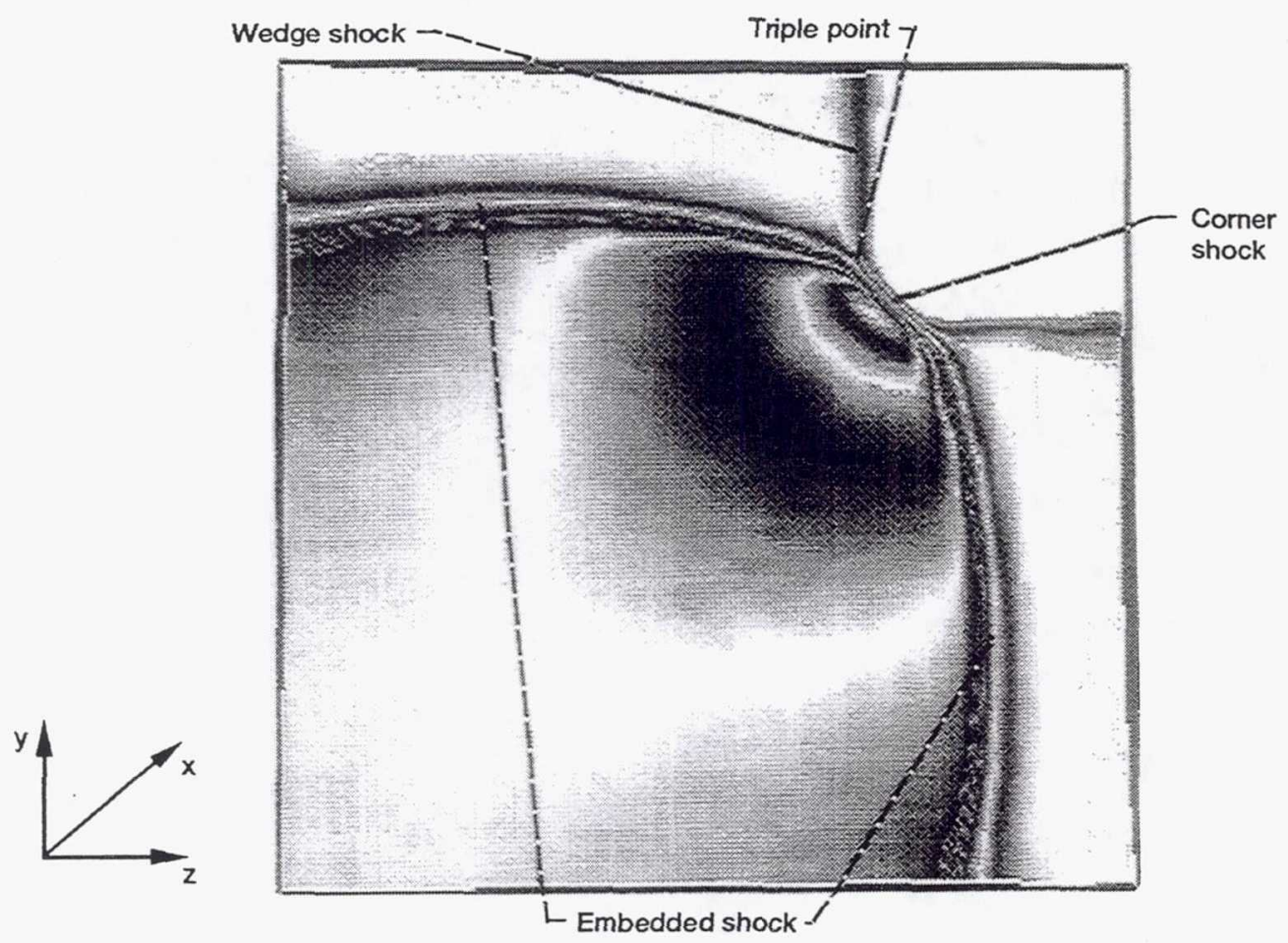

(c) Isobars.

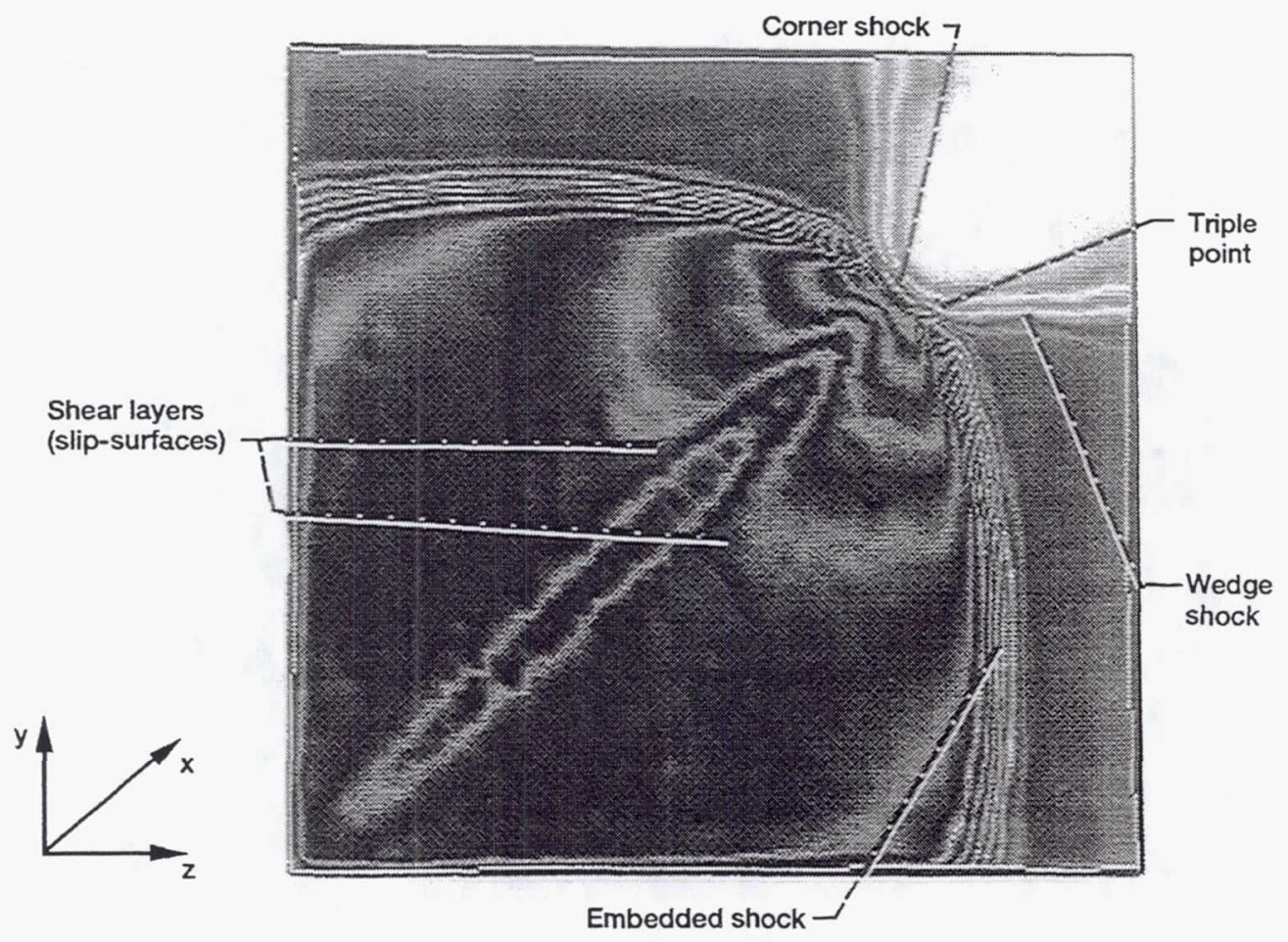

(d) Isopycnics (density contours) at a typical $\lambda$ plane.

Figure 7.-Continued. 


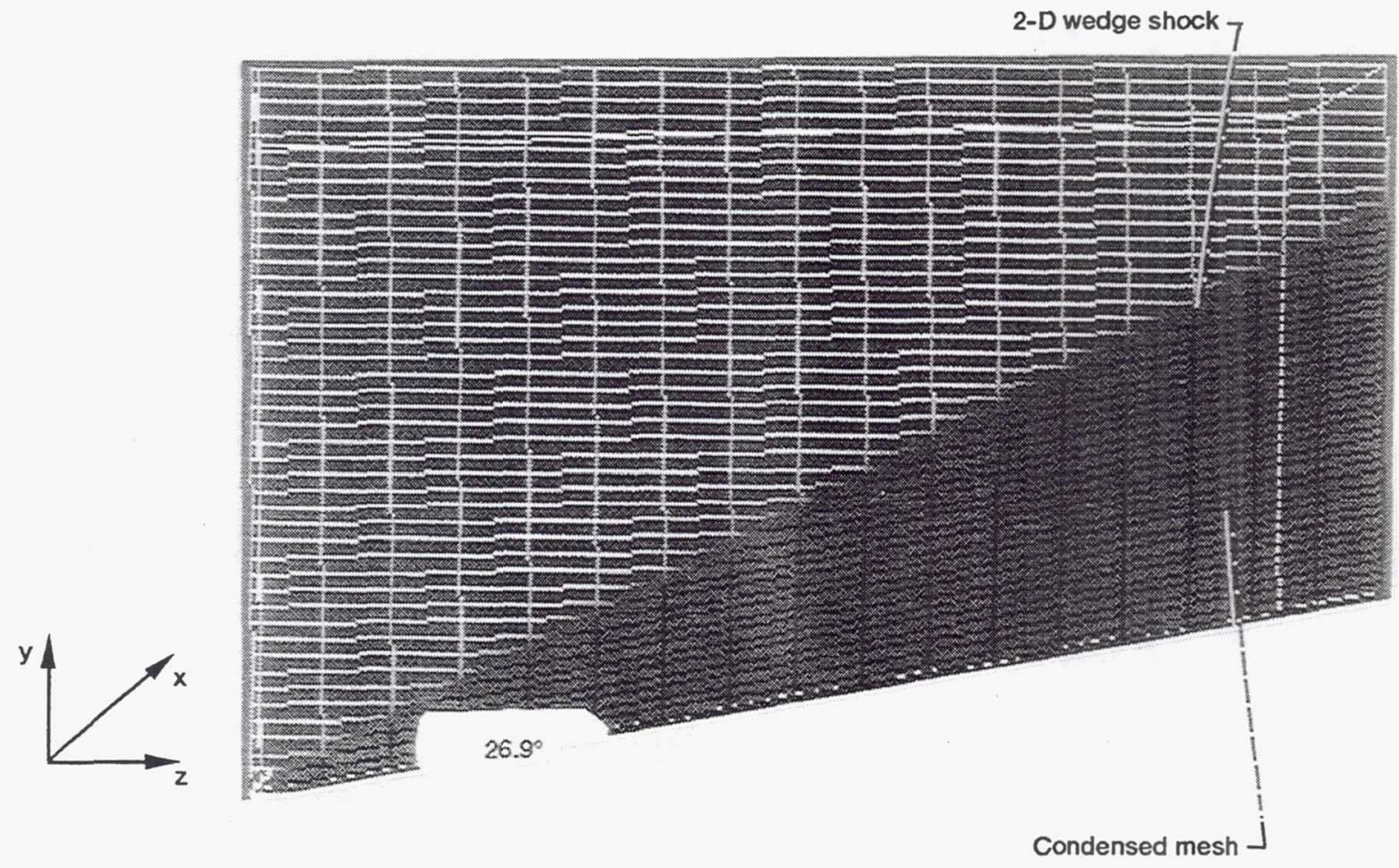

(e) Automatically generated mesh.

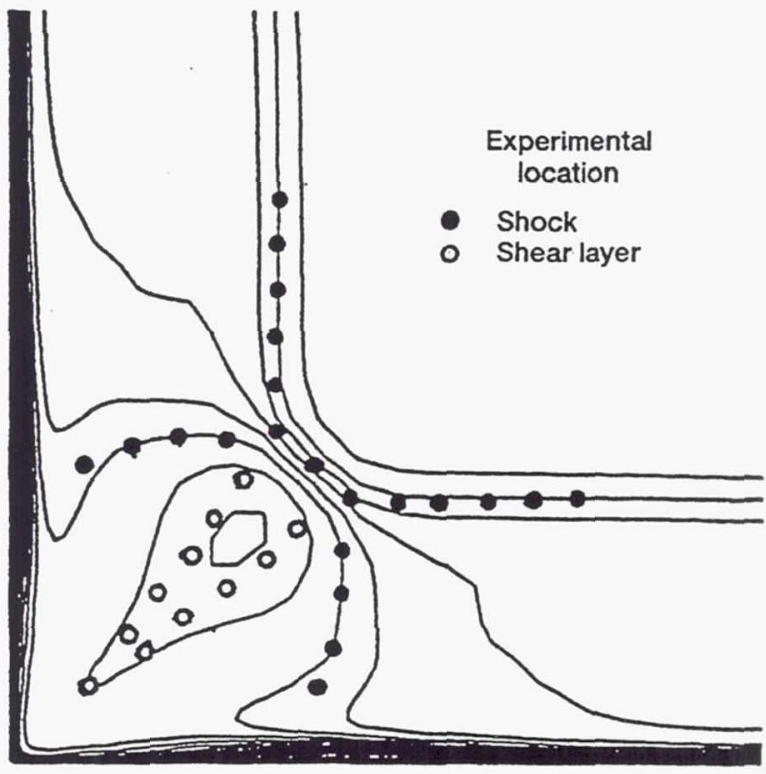

(f) Computed isomachs by Liou and Hsu (ref. 18).

Figure 7.-Concluded. 


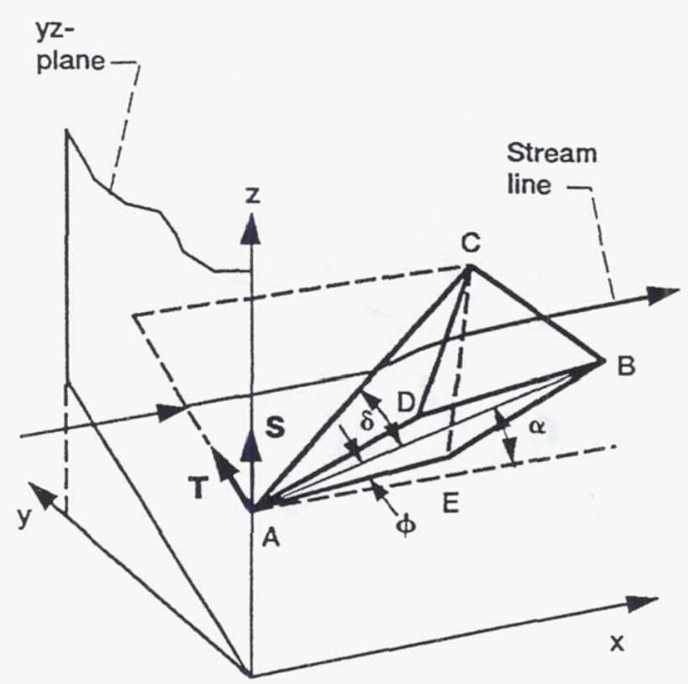

(a) Symmetrical wing body.

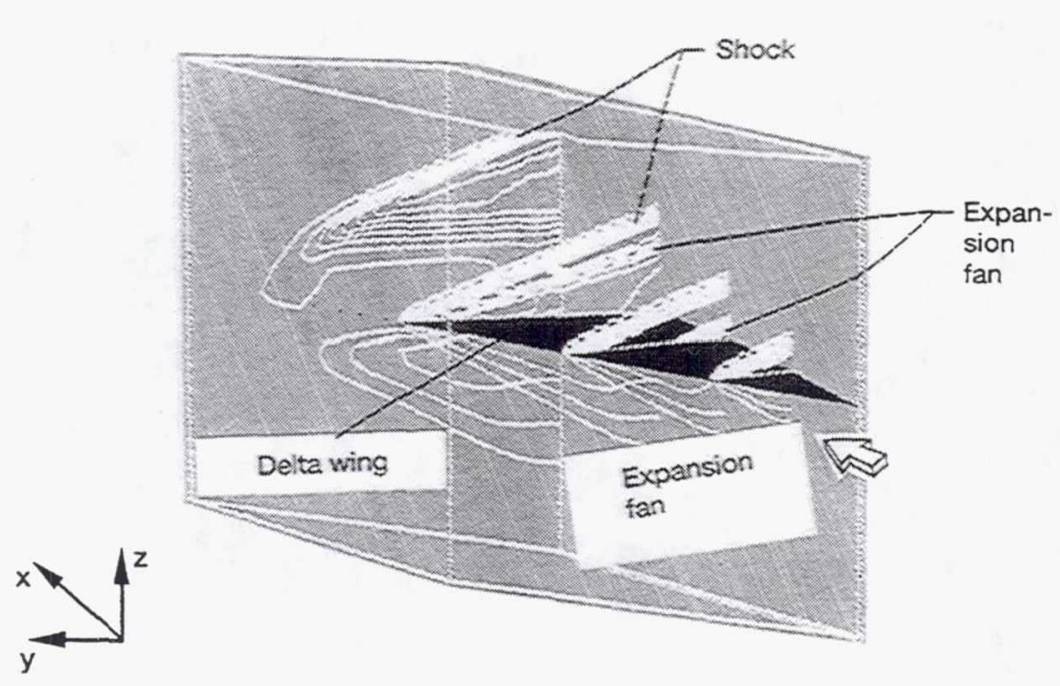

(b) Isobars at different stations around the wing body.

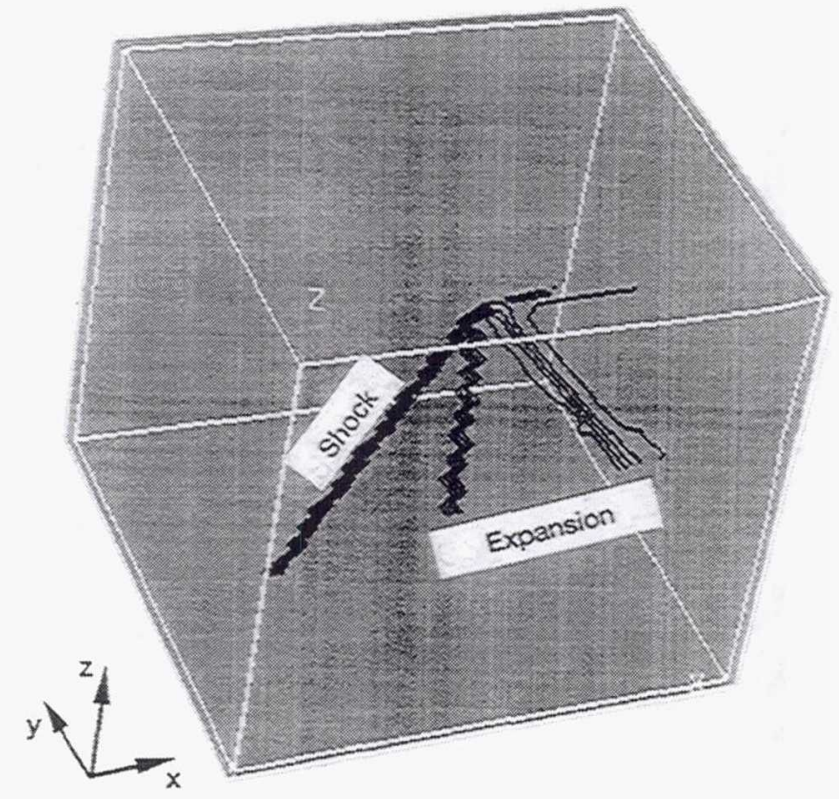

(c) isobars on the upper wing body surface.

Figure 8. -Flow of $M=4$ past a delta wing. 


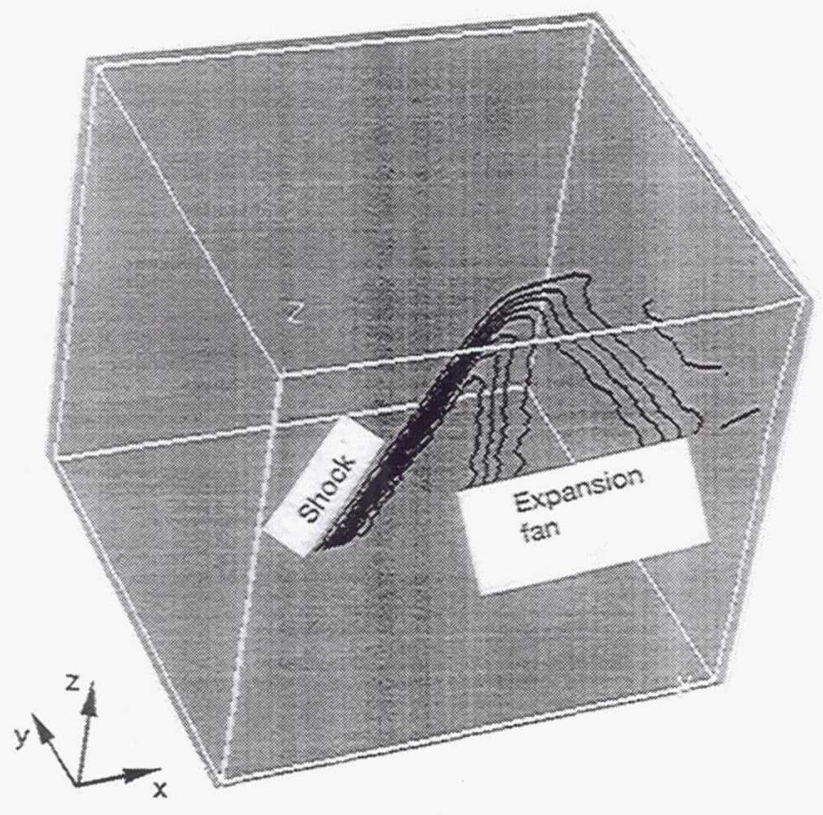

(d) Isobars at a typical stream surface above the wing body.

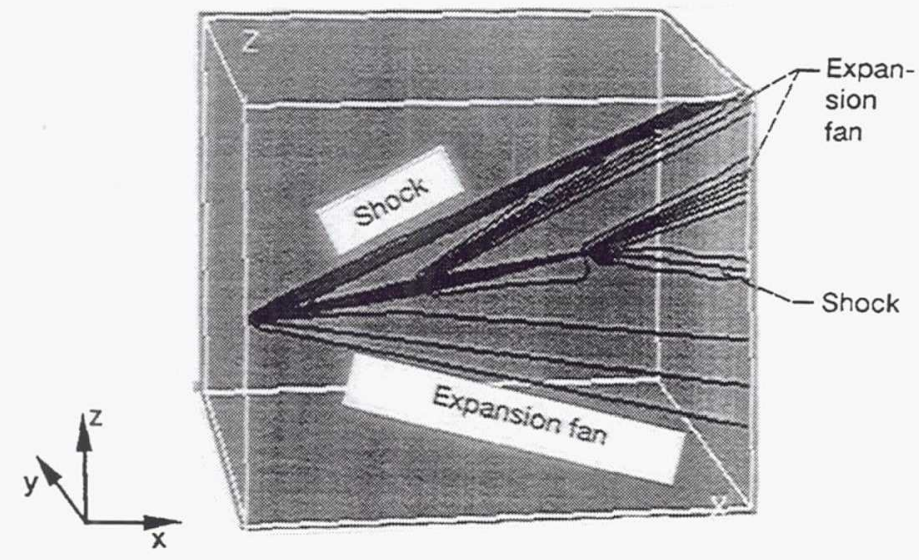

(f) Isobars at the symmetric plane.

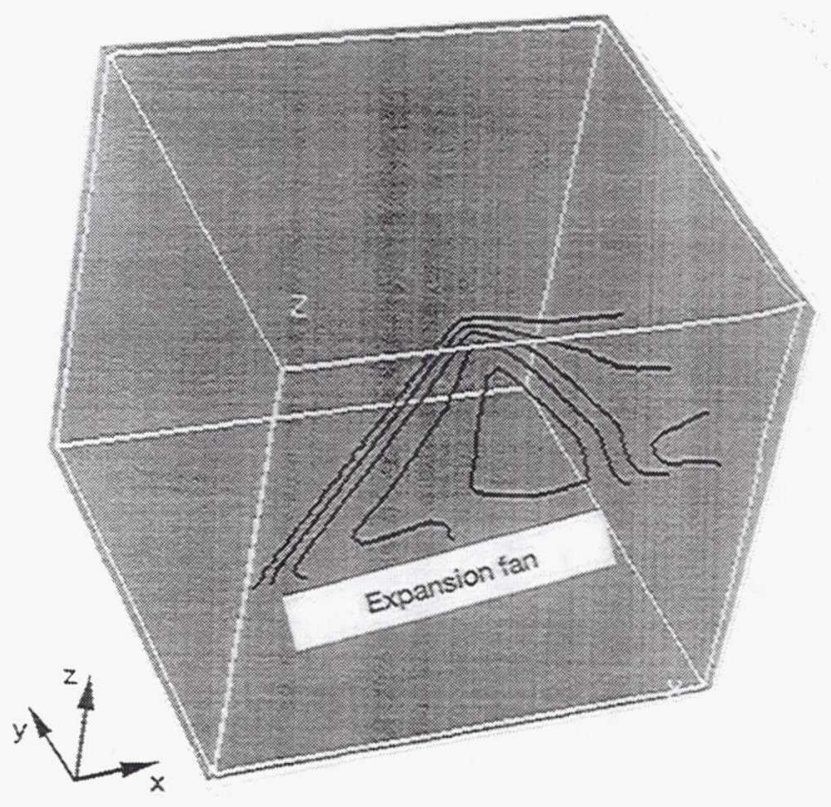

(e) Isobars at a typical stream surface beneath the wing body.

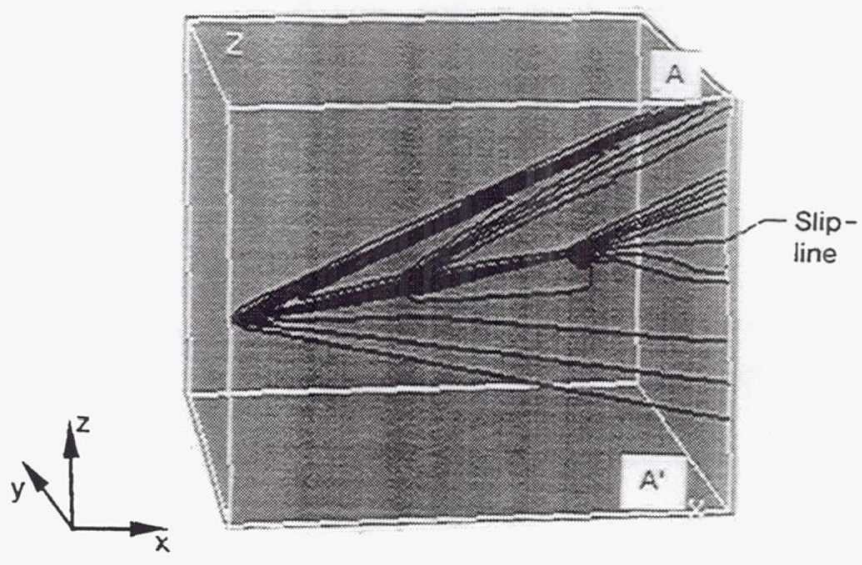

(g) Isopycnics at the symmetric plane.

Figure 8.-Continued. 


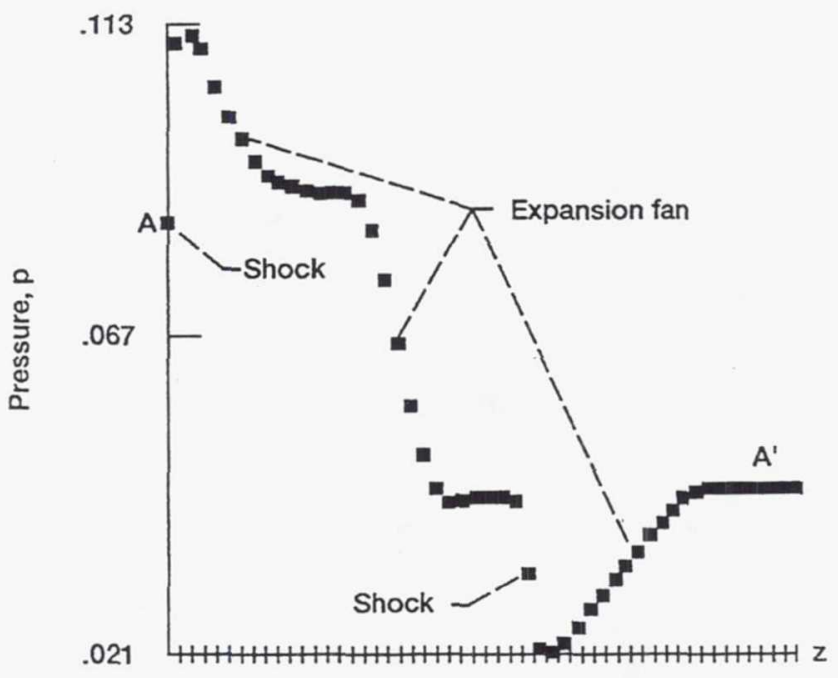

(h) Pressure distribution along a typical time line $A A^{\prime}$ at the symmetric plane.

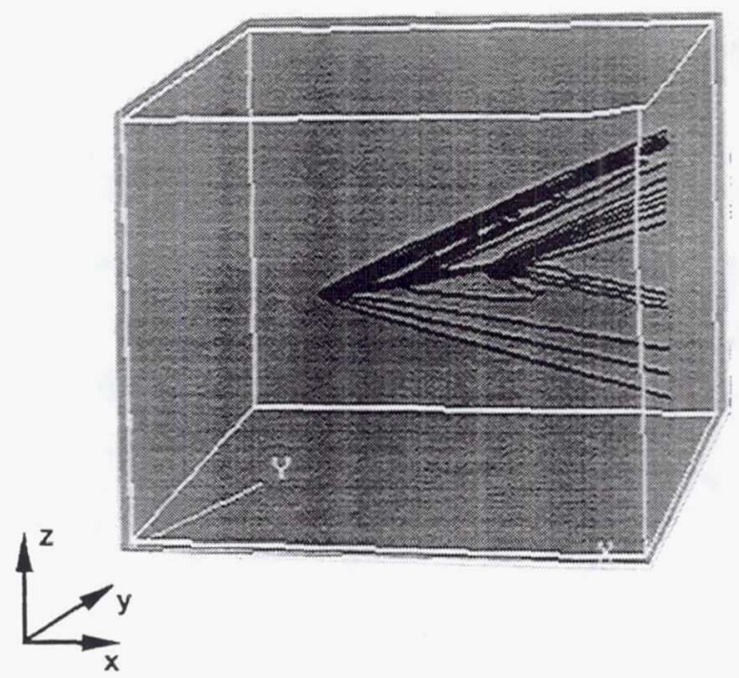

(j) Isobars at a typical stream surface parallel to the symmetric plane.

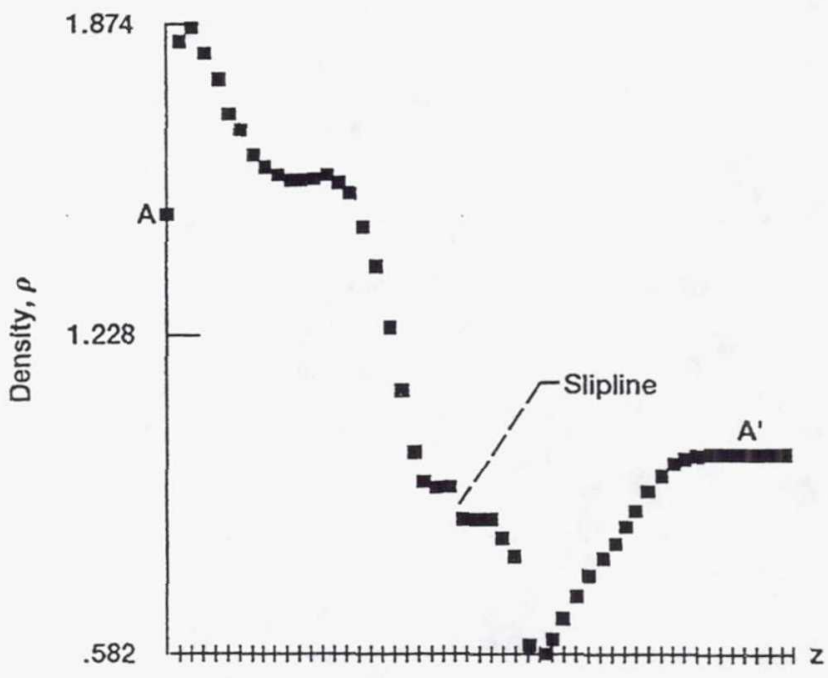

(i) Density distribution along a typical time line AA' at the symmetric plane.

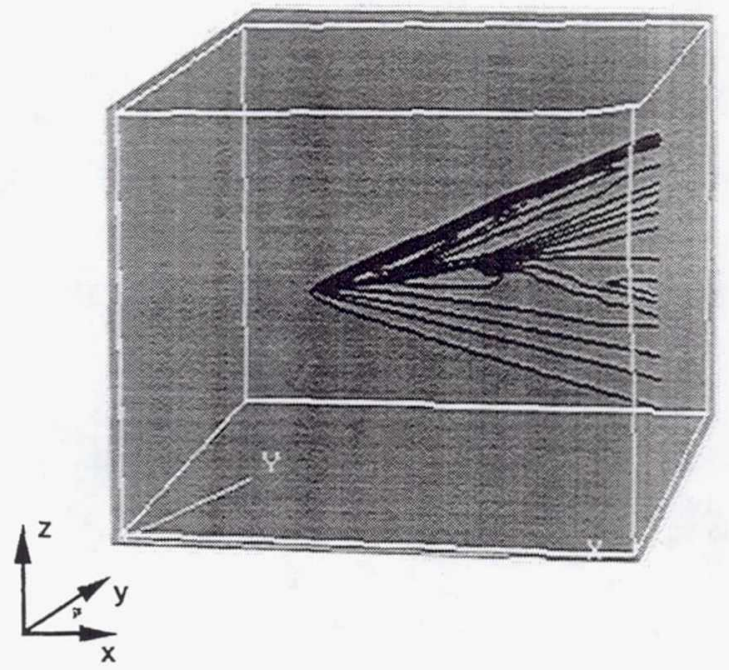

(k) Isopycnics at a typical stream surface parallel to the symmetric plane.

Figure 8.-Concluded. 


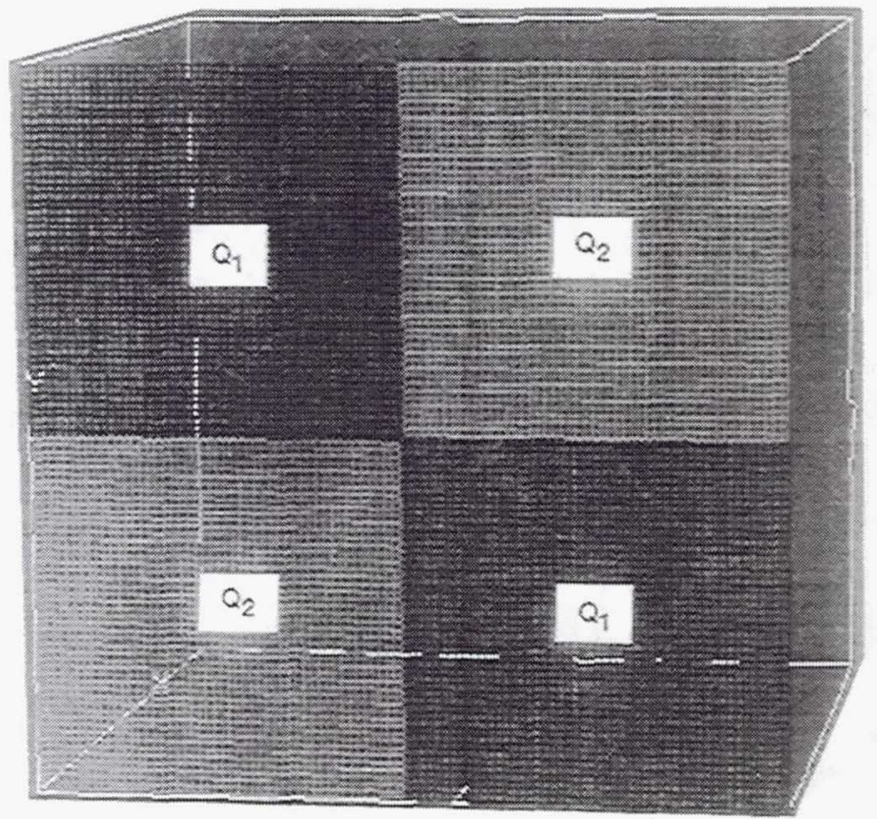

(a) Initial condition and mesh.

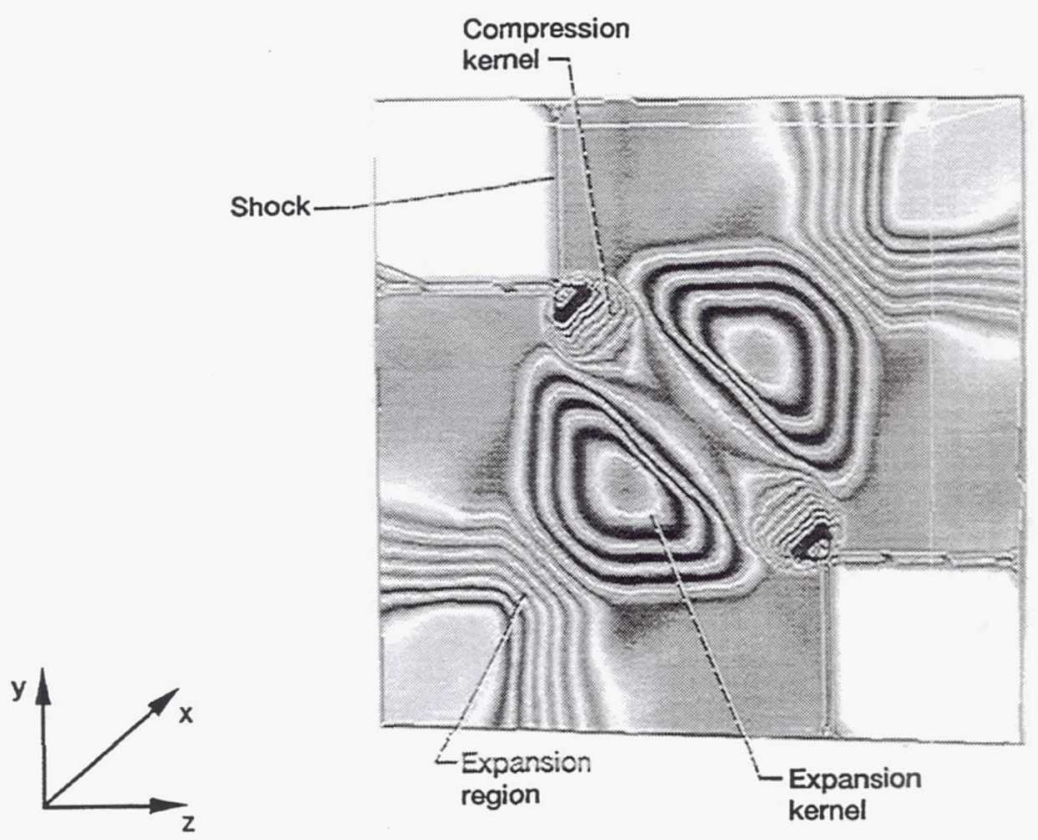

(b) Isobars.

Figure 9.-The symmetric 3-D Riemann problem. 


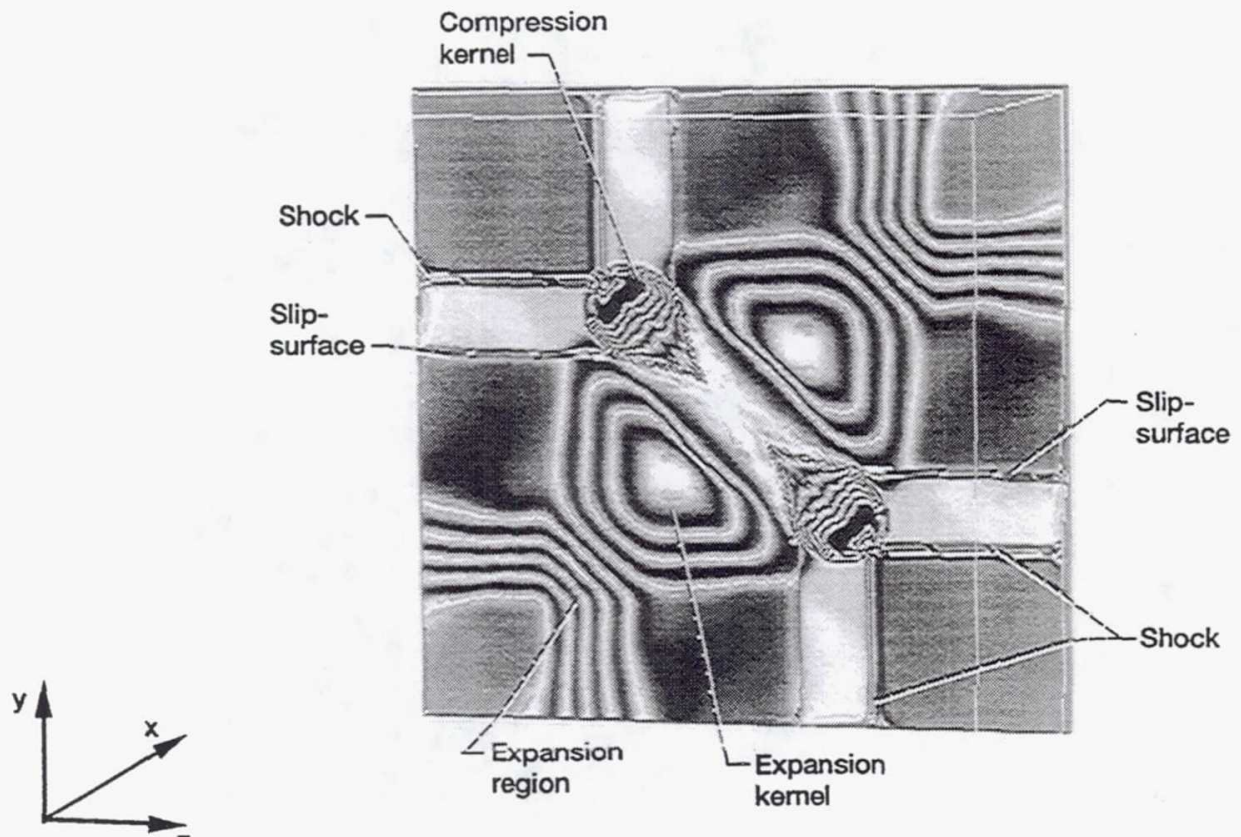

(c) Isopycnics (density contours).

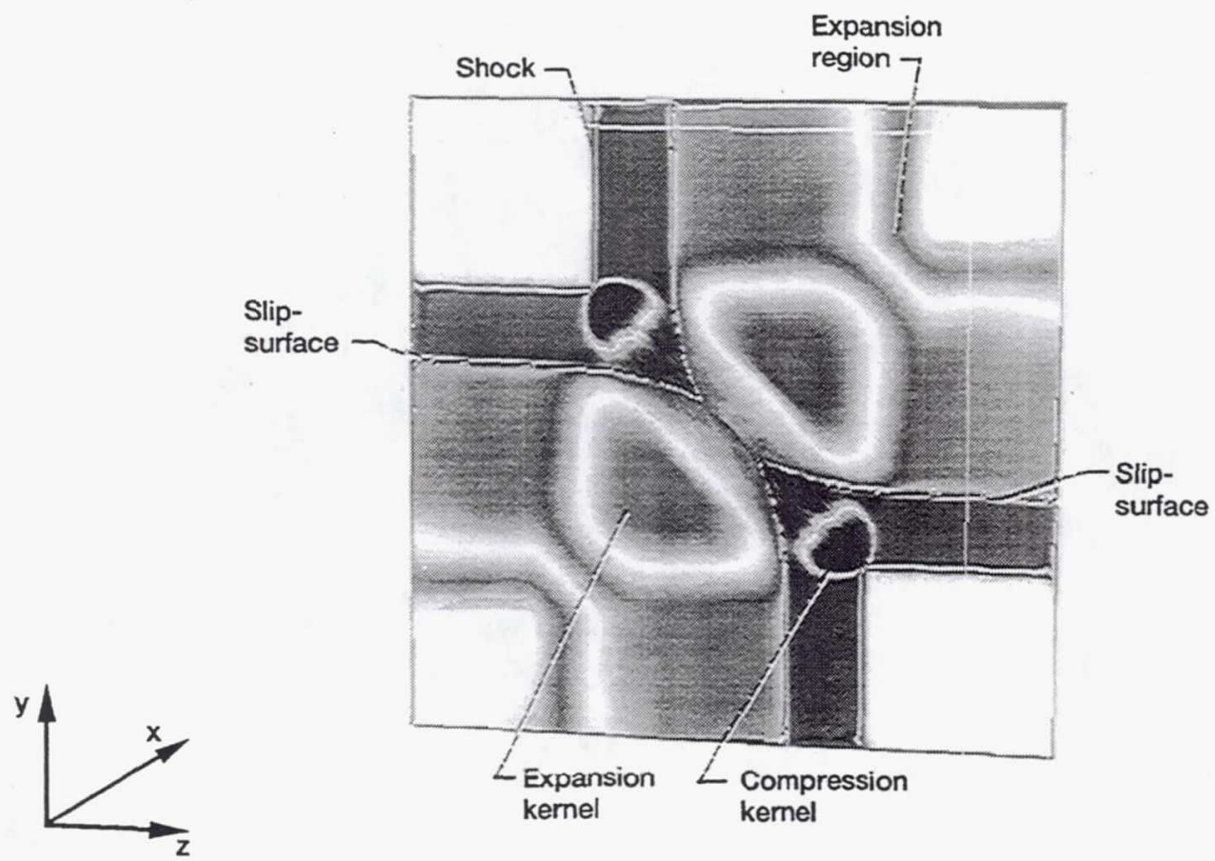

(d) U-velocity component contours on a typical distance surface ( $\lambda=$ constant).

Figure 9.-Continued. 


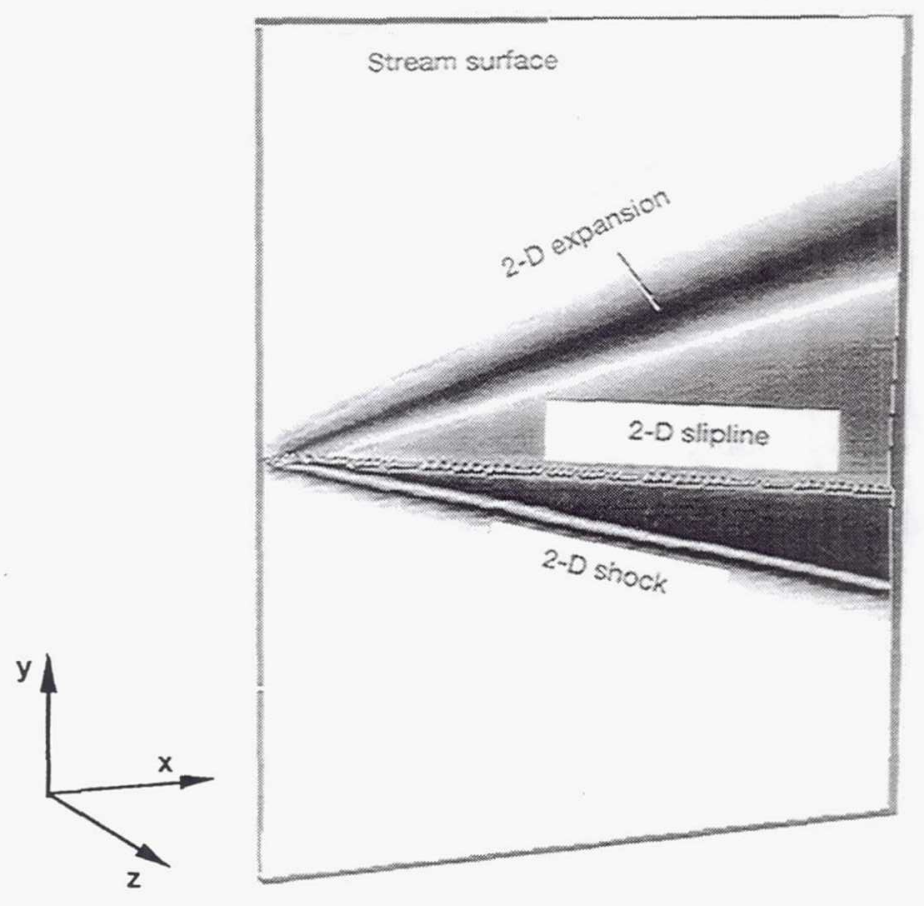

(e) Isopycnics on a side plane, showing 2-D structure.

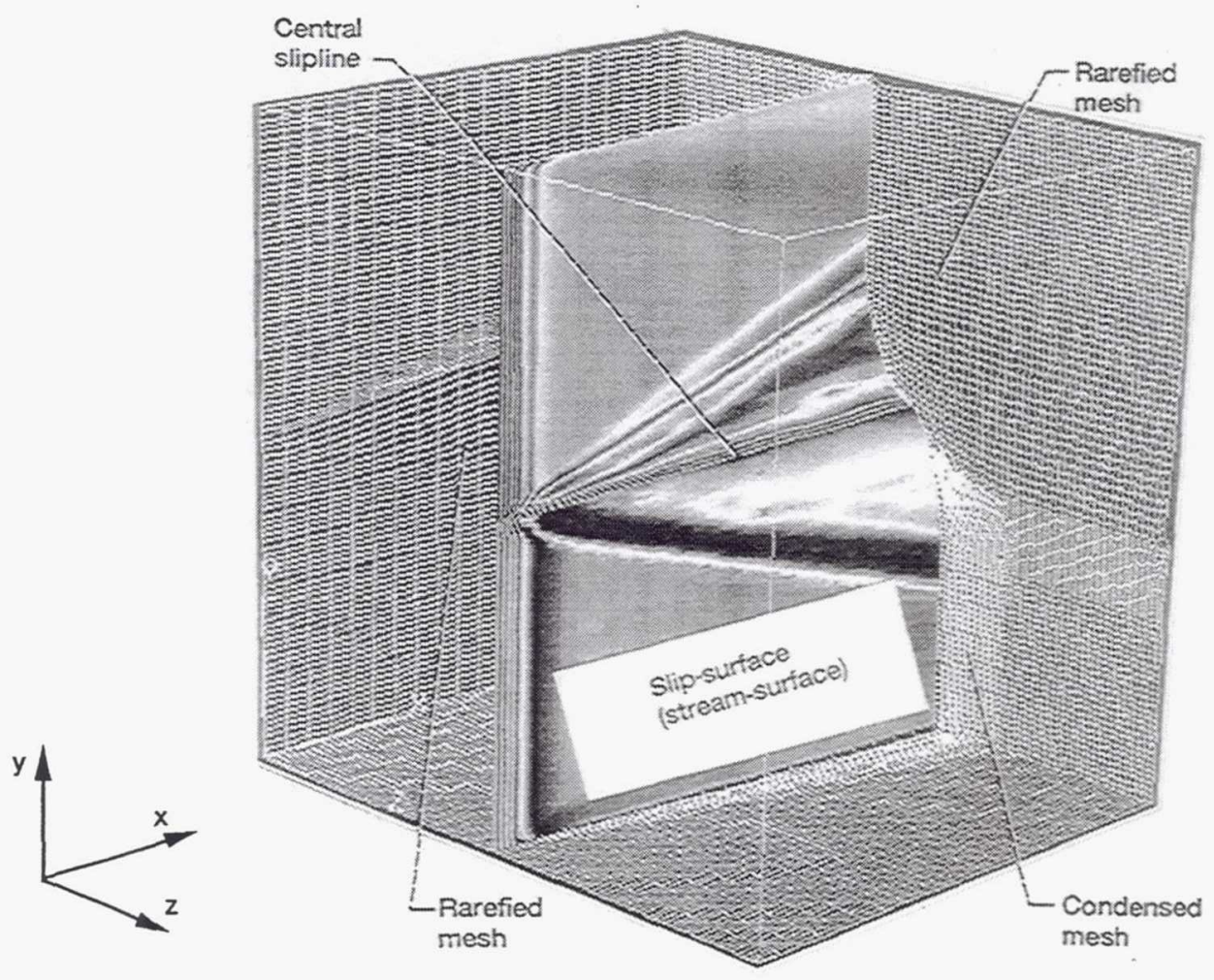

(f) Central slip-surface, showing deformation as $\lambda$ increases.

Figure 9.-Concluded. 
Public reporting burden for this collection of information is estimated to average 1 hour per response, including the time for reviewing instructions, searching existing data sources, gathering and maintaining the data needed, and completing and reviewing the collection of information. Send comments regarding this burden estimate or any other aspect of this collection of information, including suggestions for reducing this burden, to Washington Headquarters Services, Directorate for Information Operations and Reports, 1215 Jefferson Davis Highway, Suite 1204, Arlington, VA 22202-4302, and to the Oftice of Management and Budget, Paperwork Reduction Project (0704-0188), Washington, DC 20503.

\begin{tabular}{|l|l|l|}
\hline 1. AGENCY USE ONLY (Leave blank) & $\begin{array}{r}\text { 2. REPORT DATE } \\
\text { September } 1993\end{array}$ & $\begin{array}{r}\text { 3. REPORT TYPE AND DATES COVERED } \\
\text { Technical Memorandum }\end{array}$ \\
\hline
\end{tabular}

4. TITLE AND SUBTITLE

5. FUNDING NUMBERS

A New Lagrangian Method for Three-Dimensional Steady Supersonic Flows

6. AUTHOR(S)

Ching-Yuen Loh and Meng-Sing Liou

WU-505-62-52

National Aeronautics and Space Administration

Lewis Research Center

Cleveland, Ohio 44135-3191

8. PERFORMING ORGANIZATION REPORT NUMBER

E-7673

9. SPONSORING/MONITORING AGENCY NAME(S) AND ADDRESS(ES)

10. SPONSORING/MONITORING AGENCY REPORT NUMBER

National Aeronautics and Space Administration

Washington, D.C. 20546-0001

NASA TM-106068

11. SUPPLEMENTARY NOTES

Ching-Yuen Loh, National Research Council-NASA Research Associate. Meng-Sing Liou, NASA Lewis Research Center. Responsible person, Ching Y. Loh, (216) 433-6680.

12a. DISTRIBUTION/AVAILABILITY STATEMENT

12b. DISTRIBUTION CODE

Unclassified - Unlimited

Subject Category 02

13. ABSTRACT (Maximum 200 words)

In this report, the new Lagrangian method introduced by Loh and Hui is extended for three-dimensional, steady supersonic flow computation. The derivation of the conservation form and the solution of the local Riemann solver using the Godunov and the high-resolution TVD (total variation diminished) scheme is presented. This new approach is accurate and robust, capable of handling complicated geometry and interactions between discontinuous waves. Test problems show that the extended Lagrangian method retains all the advantages of the two-dimensional method (e.g., crisp resolution of a slip-surface (contact discontinuity) and automatic grid generation). In this report, we also suggest a novel threedimensional Riemann problem in which interesting and intricate flow features are present.

14. SUBJECT TERMS

Langrangian description; Three-dimensional Riemann problem;

Godunov scheme; Supersonic steady flow

17. SECURITY CLASSIFICATION OF REPORT

Unclassified
18. SECURTYY CLASSIFICATION OF THIS PAGE Unclassified
19. SECURITY CLASSIFICATION OF ABSTRACT Unclassified 